\title{
1 Revisiting Cryptococcus extracellular vesicles properties and their use as
} vaccine platforms

4 Juliana RIZZO ${ }^{1 \#}$, Sarah Sze Wah WONG ${ }^{2}$, Anastasia D. GAZI ${ }^{3}$, Frédérique MOYRAND ${ }^{1 \# \text {, }}$ 5 Thibault $\mathrm{CHAZE}^{4}$, Pierre-Henri COMMERE ${ }^{5}$, Sophie NOVAULT ${ }^{5}$, Mariette MATONDO ${ }^{4}$, 6 Gerard PEHAU-ARNAUDET ${ }^{3}$, Flavia C. G. REIS ${ }^{6,7 \#}$, Matthijn VOS $^{8}$, Lysangela R 7 ALVES $^{6 \#}$, Robin C. MAY ${ }^{9 \#}$, Leonardo NIMRICHTER ${ }^{10 \#}$, Marcio L. RODRIGUES ${ }^{6,10 \#,}$ 8 Vishukumar AIMANIANDA $^{2}$ and Guilhem JANBON ${ }^{1 \text { 1\#*}^{*}}$

${ }^{1}$ Unité Biologie des ARN des Pathogènes Fongiques, Département de Mycologie, Institut Pasteur, F75015, Paris, France

${ }^{2}$ Unité Mycologie Moléculaire, CNRS UMR2000, Département de Mycologie, Institut Pasteur, F75015, Paris, France

${ }^{3}$ Ultrastructural Bio-Imaging, UTechS UBI, Département de Biologie cellulaire et infection, UMR 3528 CNRS, Institut Pasteur, F-75015, Paris, France

${ }^{4}$ Plateforme Protéomique, Unité de Spectrométrie de Masse pour la Biologie (MSBio), Centre de Ressources et Recherches Technologiques (C2RT), UMR 2000 CNRS, Institut Pasteur, Paris, France ${ }^{5}$ Cytometry and Biomarkers, Centre de Ressources et Recherches Technologiques (C2RT), Institut Pasteur, F-75015, Paris, France

${ }^{6}$ Instituto Carlos Chagas, Fundação Oswaldo Cruz (FIOCRUZ), Curitiba, Brazil

${ }^{7}$ Centro de Desenvolvimento Tecnologico em Saude (CDTS-Fiocruz), Brazil

${ }^{8}$ NanoImaging Core Facility, Centre de Ressources et Recherches Technologiques (C2RT), Institut Pasteur, F-75015, Paris, France

${ }^{9}$ Institute of Microbiology and Infection and School of Biosciences, University of Birmingham, Birmingham B15 2TT, United Kingdom

${ }^{10}$ Instituto de Microbiologia Paulo de Góes (IMPG), Universidade Federal do Rio de Janeiro, Rio de Janeiro, Brazil

${ }^{\#}$ Members of the data sharing transparency group on fungal extracellular vesicles

* Corresponding author: janbon@ pasteur.fr 


\section{Abstract}

34

35

36

37

38

39

40

41

42

43

44

45

46

47

48

49

50

52

53

54

55

56

57

Whereas extracellular vesicle (EV) research has become commonplace in different biomedical fields, this field of research is still in its infancy in mycology. Here we provide a robust set of data regarding the structural and compositional aspects of EVs isolated from the fungal pathogenic species Cryptococcus neoformans, $C$. deneoformans and C. deuterogattii. Using cutting-edge methodological approaches including cryogenic electron microscopy and cryogenic electron tomography, proteomics, and flow cytometry, we revisited cryptococcal EV features and suggest a new EV structural model, in which the vesicular lipid bilayer is covered by mannoprotein-based fibrillar decoration, bearing the capsule polysaccharide as its outer layer. About $10 \%$ of the EV population is devoid of fibrillar decoration, adding another aspect to EV diversity. By analyzing EV protein cargo from the three species, we characterized the typical Cryptococcus EV proteome. It contains several membrane-bound protein families, including some Tsh proteins bearing a SUR7/PalI motif. The presence of known protective antigens on the surface of Cryptococcus EVs, resembling the morphology of encapsulated virus structures, suggested their potential as a vaccine. Indeed, mice immunized with EVs obtained from an acapsular $C$. neoformans mutant strain rendered a strong antibody response in mice and significantly prolonged their survival upon $C$. neoformans infection.

Keywords: Cryptococcus, fungal infections, extracellular vesicles, mannoproteins, vaccine, Cryo-EM 


\section{Introduction}

All living organisms release lipid bilayer-delimited particles defined as extracellular vesicles (EVs) (Deatherage and Cookson 2012, Witwer and Théry 2019). EV size ranges from $20 \mathrm{~nm}$ to close to one micrometer in diameter. In mammalian cells, two major classes of EVs have been defined, microvesicles and exosomes, according to their size and cellular origin (Meldolesi 2018, van Niel et al. 2018). In these organisms, a large body of literature describes how EVs participate in intercellular signaling within and in organism-to-organism communication, including carcinogenesis and host-pathogen interactions (Xu et al. 2018, Shopova et al. 2020). In fungi, the first report of fungal EVs was published in 2007 (Rodrigues et al. 2007), and, since then, their existence has been reported in many species of pathogenic and nonpathogenic fungi (Rizzo et al. 2020).

By analogy with mammalian EVs, it has been hypothesized that fungal EVs could also participate in many biological processes (Rodrigues and Casadevall 2018). Indeed, some reports indicate their relevance in diverse mechanisms related to fungal pathophysiology, such as antifungal resistance and biofilm formation (Leone et al. 2018, Zarnowski et al. 2018), transfer of virulence-associated molecules and modulation of host cells (Oliveira et al. 2010, Vargas et al. 2015, Rizzo et al. 2017, Bielska et al. 2018, Souza et al. 2019, Hai et al. 2020), cell wall remodeling and biogenesis (Zhao et al. 2019, Rizzo et al. 2020), among others (Bielska and May 2019, Rizzo et al. 2020). Nevertheless, the molecular mechanisms implicated in these exchanges of information, as well as the genetics regulating fungal EV biogenesis and release, remain elusive.

As with their mammalian, bacterial and plant counterparts, fungal EVs have been shown to contain proteins, pigments, nucleic acids, polysaccharides, lipids, among other molecules (Eisenman et al. 2009, Vallejo et al. 2012, da Silva et al. 2015, Rodrigues et al. 2015, Joffe et al. 2016, Rayner et al. 2017, Reis et al. 2021). Besides the claudin-like Sur7 family proteins, recently suggested as EV protein markers in Candida albicans (Dawson et al. 2020), no other fungal EV specific molecular marker has been reported. Indeed, the laborious and inefficient EV isolation protocols that have been used until recently have strongly limited the knowledge on their composition. Additional hurdles regarding purification methods potentially affect an accurate vesicular compositional characterization (Théry et al. 2018), including potential carryover contaminants such as protein aggregates (Chiou et al. 2018). Regarding EV morphological diversity, previous studies have reported the heterogeneity of fungal EV size, as recently reviewed (Bielska and May 2019). However, single particle analyzers such as the widely used Nanoparticle Tracking Analysis (NTA) and most common 
flow cytometers cannot reliably evaluate particles smaller than $100 \mathrm{~nm}$ in diameter (Maas et al. 2015, Théry et al. 2018, Chiang and Chen 2019, Noble et al. 2020). Overall, although a considerable number of fungal EV-related studies have been published in recent years, our knowledge of fungal EV structure and composition remains limited, which prevents further robust analysis of their functions.

Seven species of pathogenic Cryptococcus have been described (Hagen et al. 2015). Whereas species belonging to the neoformans clade (C. neoformans and C. deneoformans) typically affect immunocompromised patients, species belonging to the gattii clade (C. gattii, C. deuterogattii, C. tetragattii, C. decagattii, and C. bacillisporus) are often primary pathogens and can cause aggressive pulmonary infections as well as meningoencephalitis (Kwon-Chung et al. 2014, Rajasingham et al. 2017, Janbon et al. 2019). C. neoformans has historically been one of the most studied fungi regarding EV biology (Rodrigues et al. 2007, Bielska and May 2019, Rizzo et al. 2020). However, the only structural analyses of EVs from this organism are now very outdated and technologies used have shown tremendous improvements since then (Emelyanov et al. 2020, Noble et al. 2020).

To date, Cryptococcus EV proteomic approaches have identified 92 and 202 proteins, with very poor overlap and no evaluation of their abundance or enrichment (Rodrigues et al. 2008, Wolf et al. 2014). Finally, although the current model of fungal EV structure contains proteins located on the vesicular surface (by analogy with the mammalian EV structures (Emelyanov et al. 2020, Noble et al. 2020)), more experimental evidence is necessary to identify these putative membrane-associated molecules. Since many immunogenic proteins are often found to be associated with EVs, their vaccine potential has been explored mostly for bacterial and parasitic infections (Coakley et al. 2017, Wang et al. 2018), and more recently also for fungal infections (Colombo et al. 2019, Vargas et al. 2020).

In the present study, we used the recently described protocol (Reis et al. 2019) to obtain EV-enriched samples from Cryptococcus, together with cutting edge methodological approaches to revisit Cryptococcus EV structure, cargo, and their biological functions. Here we report a detailed analysis of three species ( $C$. neoformans, $C$. neoformans and $C$. deuterogattii) for which both a good genome annotation and RNA-Seq data were available (Janbon et al. 2014, Gonzalez-Hilarion et al. 2016, Gröhs Ferrareze et al. 2021). We produced a robust set of data containing cryo-electron microscopy (cryo-EM) and cryo-electron tomography (cryo-ET) proteomics, and nanoscale flow cytometry analysis, suggesting a new EV structural model, in addition to a list of cryptococcal EV protein markers. Our results led 
us to further evaluate the EV biological roles in murine models, emphasizing their potential use as an anti-cryptococcosis vaccine.

\section{Material and Methods}

\section{Strains and media}

The wild type strains used in the study were C. neoformans strain KN99 $\alpha$, C. deneoformans strain JEC21, C. deuterogattii strain R265, C. albicans strain SC5314, and S. cerevisiae strain S288C. The C. neoformans strain NE367 (MATa cap59A::NAT) has been previously

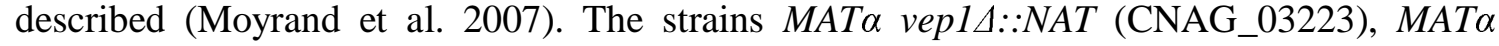

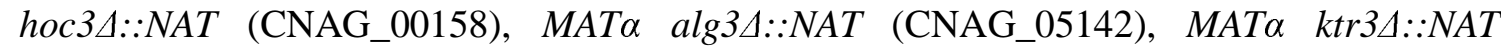
(CNAG_03832) have been constructed in the Hiten Madhani lab (UCSF, USA) and obtained from the Fungal Genetic Stock Center. To construct the strains NE1281 (MAT $\alpha$

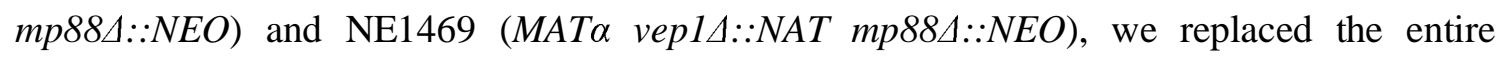
CNAG_00776 (MP88) CDS by the NEO marker in the strains KN99 $\alpha$ and MAT $\alpha$ vep1A::NAT, respectively. We here followed the previously described CRISPR CAS9 method (Fan and Lin 2018). The plasmid pPZP-NEO1 used to amplify the NEO selective marker was kindly provided by Dr. Joseph Heitman (Duke University). The deletion cassettes was constructed using a strategy previously applied to Neurospora crassa (Collopy et al. 2010). The transformants were then screened for homologous integration, as previously described (Moyrand et al. 2007). Two representatives independently obtained mutant strains were stocked at $-80^{\circ} \mathrm{C}$. All primer sequences used are provided in Table S1. All strains were taken from the G. Janbon laboratory collection at $-80^{\circ} \mathrm{C}$, plated on yeast extract-peptone-dextrose (YPD) and incubated at $30^{\circ} \mathrm{C}$ for $48 \mathrm{~h}$ before each experiment.

\section{EV isolation, labelling and proteinase $\mathrm{K}$ treatment}

EV purification was based on the recently published protocol (Reis et al. 2019) with some modifications. One loop of cells was inoculated into $10 \mathrm{~mL}$ of liquid YPD and incubated at $30^{\circ} \mathrm{C}$ for $24 \mathrm{~h}$ with shaking $(150 \mathrm{rpm})$. Cells were washed twice with $10 \mathrm{ml}$ of sterile water, counted and diluted to a density of $3.5 \times 10^{7}$ cells $/ \mathrm{mL}$ in water. Aliquots of 300 $\mu \mathrm{L}$ of the cellular suspension were spread onto synthetic dextrose (SD) solid medium plates and incubated for $24 \mathrm{~h}$ at $30^{\circ} \mathrm{C}$ to reach cell confluence. The cells were recovered from each plate with a $10 \mu \mathrm{L}$ inoculation loop and transferred to an ultracentrifugation tube containing 
$10 \mathrm{~mL} 0.22 \mu \mathrm{m}$-filter sterile of 0.01 M PBS. Cells were homogenized and collected by centrifugation at $5,000 \times \mathrm{g}$ for $15 \mathrm{~min}$ at $4 \square \mathrm{C}$. Supernatants were collected and centrifuged again at $15,000 \mathrm{x}$ g for $15 \mathrm{~min}$ at $4 \square \mathrm{C}$ to eliminate cellular debris. The resting supernatants were filtered through $0.45 \mu \mathrm{m}$ syringe filters and ultracentrifuged at $100,000 \mathrm{x}$ g for $1 \mathrm{~h}$ at $4{ }^{\circ} \mathrm{C}$ (SW41 Ti swinging-bucket rotor, Beckman Coulter). The supernatant was discarded and the pellet suspended in $0.22 \mu \mathrm{m}$-pore filtered or $0.02 \mu \mathrm{m}$-pore filtered (for Flow Cytometry analysis) PBS for immediately use or stored at $-80^{\circ} \mathrm{C}$ for further experiments. The amount of total sterol in the EV samples was measured by the Amplex ${ }^{\mathrm{TM}}$ Red Cholesterol Assay Kit (ThermoFisher, A12216) and adjusted for the subsequent experiments.

EVs were labelled either with the Concanavalin A (ConA) - Alexa Fluor ${ }^{\mathrm{TM}} 488$ conjugated, or with the Alexa 488 labelled anti-GXM monoclonal antibody 18B7 (Casadevall et al. 1992), a kind gift of Oscar Zaragoza. The ConA stock solution (5mg/mL) was previously centrifuged at $13.000 \mathrm{x} \mathrm{rpm}$ for $2 \mathrm{~min}$, in order to eliminate possible aggregates, and diluted to $500 \mu \mathrm{g} / \mathrm{mL}$ in filtered PBS. In $1.5 \mathrm{~mL}$ Eppendorf tubes, $5 \mu \mathrm{L}$ of ConA (500 $\mu \mathrm{g} / \mathrm{mL})$, together with $5 \mu \mathrm{L} \mathrm{EV}$ suspension were add to a final volume of $100 \mu \mathrm{L}$ filtered PBS. The tubes were incubated for $1 \mathrm{~h}$ at $30^{\circ} \mathrm{C}$, under agitation and protected from light. After incubation, $10 \mathrm{~mL}$ of $0.02 \mu \mathrm{m}$-pore filtered PBS were added to the EV suspension and then submitted to ultracentrifugation for $1 \mathrm{~h}$ at $100000 \mathrm{x}$ g at $4^{\circ} \mathrm{C}$. The supernatant was again discarded and pellets suspended in $300 \mu \mathrm{L}$ of $0.02 \mu \mathrm{m}$-pore filtered before being transferred to BD Trucount ${ }^{\mathrm{TM}}$ Tubes (BD Biosciences) and proceeded to Flow Cytometry analysis. A similar protocol was applied for the EV labelling with the Alexa 488 labelled anti-GXM monoclonal antibody 18B7, which was diluted 20 times before adding to EV suspension.

$\mathrm{EV}$ proteinase $\mathrm{K}$ treatment was performed following the previously described protocol (Yang et al. 2021) with some modifications. Briefly, proteinase K was added to the EV suspension $(0.17 \mu \mathrm{g}$ of sterol) to a final concentration of $2 \mathrm{mg} / \mathrm{mL}$ in $0.02 \mu \mathrm{m}$-pore filtered PBS. After proteolysis for $1 \mathrm{~h}$ at $55^{\circ} \mathrm{C}$ under agitation $(300 \mathrm{rpm})$, the enzymatic reaction was stopped by the proteinase inhibitor PMSF $(1 \mathrm{mM})$ for $20 \mathrm{~min}$ at RT. Proteinase $\mathrm{K}$ treated EVs were finally submitted to ConA labelling, ultracentrifuge washed as described before and analyzed by flow cytometry. Control conditions included untreated EVs and EVs incubated only with PMSF.

\section{Flow cytometry}

EVs were analyzed and sorted on a cell sorter MoFlo Astrios (Beckman Coulter) equipped with an EQ module specifically developed to detect nanoparticles and with $488 \mathrm{~nm}$ 
and $561 \mathrm{~nm}$ lasers at $200 \mathrm{~mW}$. The sorting was carried out with a $70 \mu \mathrm{m}$ nozzle at a pressure of 60 PSI and a differential pressure with the sample of 0.3-0.4 PSI. The sheath liquid $\mathrm{NaCl}$ $0.9 \%$ (REVOL, France) was filtered on a $0.04 \mu \mathrm{m}$ filter. The analyses were on the SSC parameter of laser 561, with threshold set to $0.012 \%$ in order to have maximum 300 eps. An M2 mask was added in front of the FSC. All SSC and FSC parameters are viewed in logarithmic mode. The calibration of the machine was carried out using Megamix-Plus SSC beads from BioCytex. We used the Trucount ${ }^{\mathrm{TM}}$ Tubes to normalize the EV counting for ConA labelling, and the fluorescence of the Mab18B7 and alexa 488 conjugated, and beads Trucount $^{\mathrm{TM}}$ was measured on parameter 488-513/26. Control conditions including ultracentrifuge washed PBS, previously incubated with ConA were used to evaluate the PBS associated noise and to normalize labelling percentages. Flow Cytometry data were analyzed by FlowJo V10 Software.

\section{Nanoparticle tracking analysis (NTA)}

Quantitative determination of EV size distribution was performed by NTA, in addition to microscopic methods. Protocols that were recently established for the analysis of cryptococcal EVs were used (Reis et al. 2019). Briefly, ultracentrifugated pellets were 20- to 50 -fold diluted in filtered PBS and measured within the optimal dilution range of $9 \times 10^{7}$ to $2.9 \times 10^{9}$ particles/mL on an LM10 nanoparticle analysis system, coupled with a 488-nm laser and equipped with an SCMOS camera and a syringe pump (Malvern Panalytical, Malvern, United Kingdom). The data were acquired and analyzed using the NTA 3.0 software (Malvern Panalytical).

\section{Cryo-EM and cryo-ET}

EVs $(4 \mu \mathrm{L})$ were spotted on glow-discharged lacey grids (S166-3, EMS) and cryofixed by plunge freezing at $-180^{\circ} \mathrm{C}$ in liquid ethane using a Leica EMGP (Leica, Austria). Grids were observed either with Tecnai F20, or Titan Krios (Thermo Fisher Scientific). The Tecnai F20 (Thermo Fisher Scientific) was operating at 200kV and images were acquired under low-dose conditions using the software EPU (Thermo Fisher Scientific) and a direct detector Falcon II (Thermo Fisher Scientific).

Cryo-electron tomography was performed using $5 \mathrm{~nm}$ protein-A gold particles (UMC, Utrecht). These were mixed with the sample to serve as fiducial markers for subsequent image alignment. EV sample (4 $\mu \mathrm{L})$ was applied to glow discharged Lacey grids (S166-3, EMS) prior plunge-freezing (EMGP, Leica). Initial bi-directional tilt series acquired using a 
227 TECNAI F20 transmission electron microscope (FEI) operated at 200kV under parallel beam 228 conditions using a Gatan 626 side entry cryoholder. The SerialEM software (Mastronarde 2292005 , Schorb et al. 2019) was used to automatically acquire images every $2^{\circ}$ over a $\pm 45^{\circ}$ 230 range using a Falcon II direct detector with a pixel size of $2 \AA$, using a total dose of 180 231 electrons per Å2. At least 100 EV cryo-EM images obtained from TECNAI F20 were used for 232 measuring EV diameter and decoration thickness in wild type (WT) and mutant strains. For 233 each EV, an average of three different measurements were used to calculate the diameter 234 (delimited by the lipid bilayer) and the decoration thickness.

235 Dose-symmetric tilt series were collected on a 300kV Titan Krios (Thermo Scientific) 236 transmission electron microscope equipped with a Quantum LS imaging filter (Gatan, slit 237 with $20 \square \mathrm{eV}$ ), single-tilt axis holder and $\mathrm{K} 3$ direct electron detector (Gatan). Tilt series with 238 an angular increment of $2^{\circ}$ and an angular range of $\pm 60^{\circ}$ were acquired with the Tomography 239 software (Thermo Scientific). The total electron dose was between 120 and 150 electrons per $240 \AA 2$ and the pixel size at $3.38 \square \AA$. Dose symmetric tilt series were saved as separate stacks of 241 frames and subsequently motion-corrected and re-stacked from $-60^{\circ}$ to $+60^{\circ}$ using IMOD's 242 function align frames (Mastronarde and Held 2017) with the help of a homemade bash script.

243 Initial image shifts were estimated using IMOD's function tiltxcorr. Alignments were 244 further optimized in IMOD using the tracing of 30-40 gold fiducials across the tilt series. The 245 fiducial models gave an overall of a fiducial error around $6 \pm 2.7 \AA$. In cases of a higher error, 246 local alignments were taken into consideration, to further correct the sample's beam induced 247 motion observed. Three-dimensional reconstructions were calculated in IMOD by weighted 248 back projection using the SIRT-like radial filter to enhance contrast and facilitate subsequent segmentation analysis.

250

\section{EV-modeling and analysis of tomographic data}

Tomograms were displayed and analyzed using the $3 \mathrm{dmod}$ interface of IMOD (Kremer et al. 1996). EVs were modeled with manual tracing of their great circle prior the use of the spherical interpolator of IMOD. If the elliptical contours calculated could not follow the vesicular membrane adequately, further manual tracing was used before re-applying the interpolator. This involved tracing of membranes near the poles of the vesicles where the membrane information could still be followed. To evaluate and assign diameters to a total of 434 C. neoformans regular vesicles, located in 39 tomograms, the value of the perimeter of the spheroid's great circle was extracted using the imodinfo function of IMOD, from the same initial manually traced contours used for modelling. To display in $3 \mathrm{D}$ the vesicle contour data 
261 were meshed using the imodmesh function of IMOD. The projections of the 3D spheroidal models were displayed and rotated to study their 3D geometry.

For the evaluation of the decoration thickness, regular vesicles were analyzed by manually measuring the outer EV diameter (delimited by the fibrillar decoration) and the inner diameter (delimited by the lipid bilayer), across the longest axis of the vesicle. The final calculation of the decoration thickness was the subtraction of the inner diameter from the outer diameter, divided by two. For the modeling of the fibrillar decoration, the IMOD surface models were imported to UCSF Chimera (Pettersen et al. 2004). The models were used as masks to extract a slab of data around their outer surface, corresponding to the decoration. The thickness of the slab used refers to the mean value provided by the aforementioned manual analysis. Iso-surface representation of the decoration and final 3D data visualization of the models performed with UCSF Chimera (Pettersen et al. 2004).

\section{Immunization assays}

The animal experiments were approved by the ethical committee for animal experimentation Comité d'Éthique en Experimentation Animale (CETEA Project license number 2013-0055). Six-week old female BALB/c mice (Janvier Labs) were used for immunization study. The amount of EVs, in protein concentration, was determined by BCA method prior to immunization. Following, three intraperitoneal injections (fixing protein concentration in the EVs to either 1 or $10 \mu \mathrm{g}$ and suspending in $100 \mu \mathrm{L} \mathrm{PBS}$ ) at 15-day intervals were given to the mice. The control group of mice was injected only with PBS. Blood was collected from the submandibular veins of the mice three days after the last immunization and just before the fungal infection and tested for antibody response by Western blot. Briefly, the EVs-associated proteins were separated on $12 \%$ SDS-PAGE, and electroblotted to nitrocellulose membrane. By Western blotting, using the mouse sera at dilution 1:1000 and anti-mouse IgG antibody conjugated to peroxidase (Sigma Aldrich), the antibody response specific to the EVassociated proteins was examined. Once the antibody response was confirmed, all the immunized and control mice were challenged intranasally, around one month from the last immunization, with $1 \times 10^{4}$ cells of $C$. neoformans wild-type strain, and their body weights and survival were monitored until all mice succumbed to the infection. The immunization assay was performed in two biological replicates.

\section{Vesicle denaturation and protein digestion}




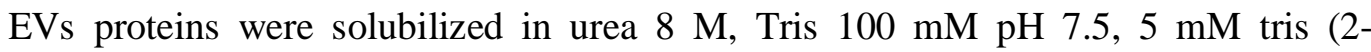

295

296

297

298

299

300

301

302

303

304

305

306

307

308

309

310

311

312

313

314

315

316

317

318

319

320

321

322

323

324

325

326

327 carboxyethyl) phosphine (TCEP) for $20 \mathrm{~min}$ at $23^{\circ} \mathrm{C}$. Samples were sonicated using a Vibracell 75186 and a miniprobe $2 \mathrm{~mm}$ (Amp 80\% // Pulse 10 off 0.8, 3 cycles). Proteins were then alkylated with $20 \mathrm{mM}$ iodoacetamide for $30 \mathrm{~min}$ at room temperature in the dark. Subsequently, LysC (Promega) was added for the first digestion step (protein to Lys-C ratio = $80: 1)$ for $3 \mathrm{~h}$ at $30^{\circ} \mathrm{C}$. Then samples were diluted down to $1 \mathrm{M}$ urea with $100 \mathrm{mM}$ Tris $\mathrm{pH} 7.5$, and trypsin (Promega) was added to the sample at a ratio of $50: 1$ for $16 \mathrm{~h}$ at $37^{\circ} \mathrm{C}$. Proteolysis was stopped by adding Formic acid (FA) to a final concentration of $1 \%$ (vol/vol). Resulting peptides were desalted using Sep-Pak SPE cartridge (Waters) according to manufactures instructions.

\section{LC-MS/MS of tryptic digest}

LC-MS/SM analysis of trypsin-digested proteins (peptides) was performed on an Orbitrap Q Exactive Plus mass spectrometer (Thermo Fisher Scientific, Bremen) coupled to an EASY-nLC 1200 (Thermo Fisher Scientific). A home-made column was used for peptide separation $\left[\mathrm{C}_{18} 40 \mathrm{~cm}\right.$ capillary column picotip silica emitter tip (75 $\mu \mathrm{m}$ diameter filled with $1.9 \mu \mathrm{m}$ Reprosil-Pur Basic $\mathrm{C}_{18}-\mathrm{HD}$ resin, (Dr. Maisch $\mathrm{GmbH}$, Ammerbuch-Entringen, Germany)]. It was equilibrated and peptide was loaded in solvent A (0.1\% FA) at 900 bars. Peptides were separated at $250 \mathrm{~nL} \cdot \mathrm{min}^{-1}$. Peptides were eluted using a gradient of solvent B (ACN, $0.1 \%$ FA) from $3 \%$ to $22 \%$ in $160 \mathrm{~min}, 22 \%$ to $50 \%$ in $70 \mathrm{~min}, 50 \%$ to $90 \%$ in $5 \mathrm{~min}$ (total length of the chromatographic run was 250 min including high ACN level step and column regeneration). Mass spectra were acquired in data-dependent acquisition mode with the XCalibur 2.2 software (Thermo Fisher Scientific, Bremen) with automatic switching between MS and MS/MS scans using a top-10 method. MS spectra were acquired at a resolution of 70000 (at $\mathrm{m} / \mathrm{z} 400$ ) with a target value of $3 \times 10^{6}$ ions. The scan range was limited from 300 to $1700 \mathrm{~m} / \mathrm{z}$. Peptide fragmentation was performed using higher-energy collision dissociation (HCD) with the energy set at $27 \mathrm{NCE}$. Intensity threshold for ions selection was set at $1 \times 10^{6}$ ions with charge exclusion of $\mathrm{z}=1$ and $\mathrm{z}>7$. The MS/MS spectra were acquired at a resolution of 17500 (at $\mathrm{m} / \mathrm{z}$ 400). Isolation window was set at $1.6 \mathrm{Th}$. Dynamic exclusion was employed within $45 \mathrm{~s}$.

\section{Data processing}

Data were searched using MaxQuant (version 1.5.3.8 and 1.6.6.0) (Cox and Mann 2008, Tyanova et al. 2016) using the Andromeda search engine (Cox et al. 2011) against 
home-made databases. The following databases were used. For C. neoformans KN99 $\alpha, C$. deneoformans JEC21 and $C$. deuterogattii R265 we used the recently updated proteomes (Wallace et al. 2020, Gröhs Ferrareze et al. 2021). The following search parameters were applied: carbamidomethylation of cysteines was set as a fixed modification, oxidation of methionine and protein N-terminal acetylation were set as variable modifications. The mass tolerances in MS and MS/MS were set to $5 \mathrm{ppm}$ and $20 \mathrm{ppm}$ respectively. Maximum peptide charge was set to 7 and 7 amino acids were required as minimum peptide length. A false discovery rate of $1 \%$ was set up for both protein and peptide levels. The iBAQ intensity was used to estimate the protein abundance within a sample (Schwanhäusser et al. 2011).

\section{Statistical analysis}

All statistical analyses were performed using GraphPad Prism 8 software (GraphPad Software Inc.). Data sets were tested for normal distribution using Shapiro-Wilk or Kolmogorov-Smirnov normality tests. In the cases in which the data passed the normality test, they were further analyzed using the unpaired Student's $t$ test or ordinary one-way ANOVA. When at least one data set was nonnormally distributed, we used the nonparametric Kolmogorov-Smirnov or Kruskal-Wallis test. For the comparison of the survival curves, we used the Logrank (Mantel-Cox) test.

\section{Results}

\section{- Diversity of cryptococcal EVs}

Several groups have performed morphological studies of fungal EVs by electron microscopy (Rodrigues et al. 2007, Oliveira et al. 2009, Rayner et al. 2017, Bleackley et al. 2020). However, most of these studies used sample fixation-dehydration procedures for transmission electron microscopy (TEM), which can often affect the size and morphology of EVs (Van Der Pol et al. 2010, Chiang and Chen 2019). Cryo-EM imaging on rapidly-frozen samples at low temperature could potentially reduce sample damaging and artifacts caused by the addition of heavy metals, dehydration, or fixation steps (Orlov et al. 2017, Chiang and Chen 2019). Indeed, diverse morphologies of EVs derived from even a single mammalian cell type have been clearly revealed under cryo-EM (Zabeo et al. 2017). We therefore used cryoEM and cryo-ET to analyze EVs purified from C. neoformans, in their near-native state. 
Based on the optimized version of the EV purification protocol recently described by

362

363

364

365

366

367

368

369

370

371

372

373

374

375

376

377

378

379

380

381

382

383

384

385

386

387

388

389

390

391

392

393

394 Reis and collaborators (Reis et al. 2019), we isolated EVs from C. neoformans reference strain KN99 $\alpha$, cultured on synthetic dextrose solid medium for $24 \mathrm{~h}$, in order to limit the carryover of potential contaminants. Cryo-ET tomograms allowed us to analyze 533 single vesicles, which were characterized according to their morphological aspects in regular (roundbilayered vesicles) and irregular (not rounded, bi- or multilayered vesicles) categories. Although a large proportion (81.4\%) of the observed EVs had the typical round shape, $18.6 \%$ corresponded to irregular morphologies. Among them, we observed examples of multilayered vesicles, long tubular, flat, short tubular and miscellaneous morphologies (Fig. S1; Table S2). However, it remains to be determined whether EVs with irregular morphologies are produced biologically or they appear as a consequence of the purification method.

Cryo-EM analysis showed a considerable polymorphism of EVs, with the two leaflets of the typical vesicular membrane readily visible for all EVs observed, and a few unstructured aggregates, thus confirming the quality of our preparation (Fig. 1A). In C. neoformans, among the regular vesicles, only $10.8 \%$ appeared to have a smooth surface (Fig. 1B and 1C); the majority of regular EVs $(89.2 \%)$ were decorated with a fibrillar structure anchored to the lipid bilayer (Fig. 1D and 1E). Strikingly, regardless of the morphology, the majority of EVs analyzed $(88.6 \%)$ appeared to be coated with this fibrillar material. We used cryo-ET to prepare a three-dimensional surface model of the EVs, using IMOD (Mastronarde and Held 2017) and UCSF Chimera (Pettersen et al. 2004) to further visualize their structure and fibrillar decoration (Fig. 1F to 1H).

Additional aspects of $C$. neoformans EV diversity, such as the distribution of size and decoration, were analyzed. NTA analyses showed a diameter size distribution from 80 to 500 $\mathrm{nm}$ and revealed a major peak of vesicle detection in the 150-nm-diameter range (Fig. 2A), in line with previous findings (Reis et al. 2019). We also analyzed the EV diameter frequency distribution by cryo-EM from 434-single regular EV captures (Fig. 2B). The size distribution of vesicles tracked with NTA was different from the distribution of vesicles observed with cryo-EM, which revealed a wider range of EV diameter size, ranging from as small as $10 \mathrm{~nm}$ to $500 \mathrm{~nm}$ (Fig. 2C; Video S1). Notably, smaller vesicles (< $100 \mathrm{~nm})$ comprised a higher proportion of vesicles captured by cryo-EM than by NTA. Although cryo-EM has some statistical limitations, it nonetheless confirms the known bias of NTA towards larger EVs (Bachurski et al. 2019).

Analysis of the EV size according to the presence or absence of the surface decoration revealed a different frequency distribution (Fig. 2D), with non-decorated EVs showing a 
395

396

397

398

399

400

401

402

403

404

405

406

407

408

409

410

411

412

413

414

415

416

417

418

419

420

421

422

423

424

425

426

427

428

significantly smaller size distribution ( $p=0.01$, using nonparametric Kolmogorov-Smirnov test) compared to the decorated ones (Fig. 2E). Additionally, the analysis of the vesicular decoration in 105 single regular EVs revealed heterogeneity in their thickness, ranging from 5 to $23 \mathrm{~nm}$ with the average value close to $16 \mathrm{~nm}$ (Fig. 2 F). There was no correlation between vesicular diameter size and decoration thickness, as indicated by linear regression analysis (Fig. 2G). Therefore, the presence or absence of decoration, and even its thickness, does not depend on the size and shape of the EVs, revealing a previously unknown aspect of fungal EV diversity.

We analyzed EVs from two other pathogenic species of Cryptococcus, $C$. deneoformans strain JEC21 and C. deuterogattii strain R265. As expected, cryo-EM revealed a similar structure of the EV population in the three Cryptococcus species, the majority of EVs being decorated in C. deneoformans (72.4\%) and C. deuterogattii (81.4\%) (Table S2). In contrast, $C$. deuterogattii EVs appeared to be smaller (median size $=48 \mathrm{~nm}$ ) than those of C. neoformans $($ median size $=67 \mathrm{~nm})$ and $C$. deneoformans $($ median size $=70 \mathrm{~nm})$ (Fig. 3A). In addition, the thickness of decoration is smaller in $C$. deneoformans and $C$. deuterogattii than in C. neoformans (Fig. 3B), suggesting a tight genetic control of these EV structural properties (Fig. 3C, Fig. S2).

\section{- Cryptococcus EVs structural analysis}

C. neoformans is an encapsulated microorganism, and its capsule is mostly composed of the polysaccharide glucuronoxylomannan (GXM), a critical virulence factor of this pathogenic yeast (O'Meara and Alspaugh 2012). GXM has been previously shown to be exported by EVs (Rodrigues et al. 2007). Therefore, we reasoned that the fibrillar decoration observed around the vesicles could be composed of GXM. We thus incubated C. neoformans EVs with the Alexa 488 labelled anti-GXM monoclonal antibody 18B7 (Casadevall et al. 1992), and analyzed the EV suspension by flow cytometry. More than $70 \%$ of the EVs obtained from the wild-type strain were recognized by this antibody (Fig. 4A), suggesting that most C. neoformans EVs are covered to some extent with GXM or derivatives thereof. While, EVs obtained from the acapsular mutant strain (cap594) (Moyrand et al. 2007) showed negligible labelling (2.33\%), following the same experimental approach (Fig. 4B). Nevertheless, cryo-EM observation of cap594 EVs revealed similar fibrils as observed in the wild type EVs (Fig. 4B). Moreover, cryo-EM analysis of EVs purified from cap594 suggested a similar percentage of decorated EVs (91.6\%). Overall, these data suggest that, 
429 even though GXM covers most $C$. neoformans EVs, the visible fibrillar structures around 430 them are not GXM-based. Cryo-EM analysis of EVs obtained from C. albicans SC5314 and 431 S. cerevisiae S288C grown on SD medium showed a similar fibrillar decoration observed 432 around Cryptococcus EVs (Fig. 4C), reinforcing the notion that this structure is not GXMbased, since neither of these two yeasts can synthesize this capsular polysaccharide.

We then reasoned that EV decoration could be protein-based and therefore performed proteomic analyses to further explore this novel fungal vesicular feature. Two proteomic analyses of $C$. neoformans EVs have been reported previously (Rodrigues et al. 2008, Wolf et al. 2014) wherein the authors identified 92 and 202 proteins associated with EVs in $C$. neoformans, respectively. However, neither quantitative nor enrichment of EV-associated proteins was performed in these two studies. Therefore, we performed EV proteomic characterization, together with an enrichment analysis in order to distinguish the proteins associated with EVs, from those related to potential carry-over aggregates, inevitably contaminating EV preparations.

In fungi, and more specifically in Cryptococcus, the relationship between RNA and protein abundances has been reported as nearly linear, due to the relatively minor contribution of posttranscriptional regulations to protein abundance (Wallace et al. 2020). We thus used cellular RNA abundance at $30^{\circ} \mathrm{C}$, exponential phase (Wallace et al. 2020), as a proxy for cellular protein abundance, and for normalization of EV proteome data. C. neoformans EVs proteomic analysis was performed in experimental triplicate that produced a common list containing 1847 proteins (Table S3). Proteins were ranked according to their prevalence in the sample evaluating the average intensity-based absolute quantification (IBAQ) value of the three replicates. We then used the gene expression level as evaluated by RNA-seq analysis to calculate an enrichment coefficient comparing the expected value in the cells with the observed one in EVs. We thus identified 39 non-ribosomal proteins which were present both within the 100 most prevalent EV proteins overall and the 100 most enriched proteins (Table S4). We considered these proteins as EV-associated proteins. Only 9 out of these 39 proteins were reported in previous proteomic analysis, emphasizing the necessity for proteomic data enrichment analysis. Of note, our study and those published before used different culture media, and distinct protocols of EV isolation, which might also explain the differences in protein composition that were presently observed.

To further explore how conserved the EV protein cargo across Cryptococcus species is, we proceeded with the same strategy to characterize the EV-associated proteins in two other cryptococcal species, C. deneoformans (strain JEC21) and C. deuterogattii (strain 
463 R265). We identified 38 and $48 \mathrm{EV}$-associated proteins for $C$. deneoformans and $C$. 464 deuterogattii, respectively (Table S3; Table S4). Overall, 71 EV-associated proteins were identified, 37 in at least two species, and 17 shared by all the three species (Fig. 5A and B), supporting a conserved profile of the EV-associated proteins across Cryptococcus species, and the robustness of our analyses. Several families of proteins appeared to be typical of Cryptococcus EVs. The major one was the Chitin deactelylase Cda family (Baker et al. 2011), composed of three members present among the $17 \mathrm{EV}$-associated proteins identified in all three Cryptococcus species analyzed. Some other families like the putative glyoxal oxidase (Gox proteins), or the Ricin-type beta-trefoil lectin domain-containing protein (Ril), have one member common to all three species EVs (i.e. Gox2 and Ril1) whereas the other members are found in only two species (Ril2 and Ril3) or are specific of one species EVs (Gox1 and Gox3) (Fig. 5C). We also identified three tetraspanin membrane proteins containing a SUR7/PalI family motif. Tsh1 and Tsh2 shared 32\% identity in their amino acid sequence. Tsh1 is present in both $C$. neoformans and $C$. deneoformans EVs whilst Tsh2 was identified in both C. neoformans and $C$. deuterogattii. The third Sur7/PalI protein shares very little sequence homology beyond the SUR7 motif and is exclusive to $C$. deuterogattii. Two Sur7 proteins have been recently identified in $C$. albicans EVs, suggesting that they might represent a common EV marker present in fungal EVs (Dawson et al. 2020). Finally, two members of the previously described pr4/barwin domain Blp protein family (Chun et al. 2011) were present in C. neoformans and $C$. deuterogattii EVs but not in $C$. deneoformans. Similarly, the two ferroxidase Cfo proteins (Jung et al. 2008) were shown to be associated only with the $C$. deuterogattii EVs but not in the two other species.

Several enzymes associated with polysaccharide degradation and modifications were present in Cryptococcus EVs. Some of these proteins are specific to one species but others are present in two or all three EV proteomes. For instance, identification within the Cryptococcus EV core proteins of Gas1 (a 1,3-beta-glucanosyltransferase), Amy1 (an alpha amylase), Exg104 (a glucan 1,3-beta-glucosidase), Hep2 (a putative heparinase) together with the Gox, Cda and Ril proteins suggest functions of EVs in cell wall processes, as previously hypothesized in S. cerevisiae (Zhao et al. 2019). We also identified the BCS-inducible membrane protein (Bim1), recently described as a critical factor for cupper acquisition in $C$. neoformans meningitis (Garcia-Santamarina et al. 2020). Finally, several of the EV proteins identified here have no predicted function; we therefore named them Vep (Vesicles enriched protein). Bioinformatics analysis of the $71 \mathrm{EV}$-associated protein sequences suggested that 
497 domain as predicted by SignalP-5.0 (Almagro Armenteros et al. 2019) and/or TMHMM v. 2.0

498 (Krogh et al. 2001), and 21 being putative GPI-anchored proteins as predicted by PredGPI

499 (Pierleoni et al. 2008), which is in good agreement with putative protein-based decoration.

500 Reflecting the general specificities of these three proteomes, the GPI-anchor EV-proteomes of

$501 C$. neoformans and C. deneoformans are nearly identical, whereas $C$. deuterogatii is more 502 diverse (Fig. 5D).

503 Mature GPI-anchored proteins can also be membrane-bound and are predicted to be 504 highly mannosylated in Cryptococcus and other fungi (Levitz et al. 2001, de Groot et al. 505 2003). We thus reasoned that these mannosylated proteins might represent the EV decorations 506 observed by cryo-EM. To test this hypothesis, we incubated EVs with ConA conjugated to 507 Alexa Fluor 488, and further analyzed by flow cytometry. Our results demonstrated that over $98.5 \%$ of vesicles were recognized by this lectin, confirming the presence of mannosylated proteins on the EV surface (Fig. 6). Similarly, EVs obtained from acapsular cap594 mutant strain also showed a high percentage of staining (95.5\%). Accordingly, EV treatment with proteinase $\mathrm{K}$ was associated with a nearly complete loss of ConA labelling of both WT and cap594 EVs (Fig. 7), overall suggesting that the outer vesicle decoration may be composed primarily of mannoproteins.

Several genes have been implicated in protein glycosylation in C. neoformans. For instance, $A L G 3$ encodes a dolichyl-phosphate-mannose-dependent $\alpha$-1,3-mannosyltransferase, deletion of which is associated with the production of truncated protein-associated neutral $\mathrm{N}$ glycans and a reduction in virulence (Thak et al. 2020). Similarly, KTR3 and HOC3 encodes $\alpha 1,2$-mannosyltransferase and $\alpha 1,6$-mannosyltransferase, respectively, regulating $O$-glycan structure and pathogenicity of $C$. neoformans (Lee et al. 2015). We reasoned that the deletion of some of these genes could alter EV production and structure. We first analyzed EV production in alg3A, hoc $3 A$, and ktr3A strains by evaluating the quantity of sterol in our EV preparations. We did not observe any significant alternation in EV production nor in the 523 percentage of ConA positive EVs in any of these deletion mutants (Fig. 8A, 8B). 524 Nevertheless, the percentage of alg3 $\triangle$ EVs labelled by ConA was slightly reduced (Fig. 8B) and alg3 $\mathrm{EV}$ decorations were less thick than wild type EVs, as revealed by cryo-EM observation (Fig. 8C, 8D; Table S2).

The two most abundant C. neoformans EV proteins, Mp88 and Vep1/CNAG_03223 are GPI-anchored and represent $23.7 \%$ of the total identified proteins. Mp88 is a basidiomycete specific protein originally identified as a major C. neoformans immunoreactive mannoprotein stimulating T cell responses (Huang et al. 2002). Vep1 (Vesicles Enriched 
531 Protein 1) is protein of unknown function sharing no homology with any C. albicans or $S$.

532 cerevisiae protein. In all three Cryptococcus species, Mp88 (Huang et al. 2002) was the most

533 prevalent EV protein. In C. deuterogattii EVs, in which the Vep1 protein is not present, Mp88

534 represents $35.4 \%$ of all EV proteins. We constructed the corresponding single and double 535 mutant strains for MP88 and VEP1 and tested their EVs for ConA binding. These mutations 536 did not strongly affect EV production although MP88 deletion was associated with a slight 537 increased production as compared to the wild type strain (Fig. 8A). However, both $m p 88 \Delta$ 538 and $m p 884$ vep $1 \triangle \mathrm{EVs}$ displayed a limited but statistically significant reduction of the ConA539 bound EVs as compared to EVs from wild-type strain (Fig. 8B). Accordingly, cryo-EM 540 analysis of $m p 88 \triangle \mathrm{EVs}$ revealed an associated reduction of the decoration thickness (Fig.8C, 541 8D) without any change in EV size distribution (Fig. 8C; Table S2; Fig. S2) suggesting that 542 cryptococcal EVs might bear a highly complex decoration, probably formed from a dynamic 543 combination of mannoproteins.Combining all these data, we propose a model for cryptococcal 544 EV structure, in which, EVs are decorated by mannosylated proteins and covered by GXM 545 (Fig. 9).

Proteomic analysis of the C. neoformans EVs identified many immunogenic proteins, including Mp88, the members of Gox and Cda families and some Vep proteins previously tested as vaccine candidates against cryptococcosis (Specht et al. 2017, Hester et al. 2020). Moreover, some of these proteins were also found to be enriched in $C$. deneoformans and $C$. deuterogattii EVs (Mp88, Cda1, Cda2, Cda3, and Gox2), suggesting that secretion of these immunogenic molecules via EVs could be a conserved feature across different species. Taking into account that cryptococcal EVs have been shown to be immune modulators (Freitas et al. 2019) and may impact the pathophysiology of the infection (Bielska et al. 2018, Hai et al. 2020), we reasoned that EVs could be used for immunization against cryptococcosis, avoiding the need for recombinant protein purification and adjuvant usage.

558 The usage of fungal EVs has been previously suggested as a promising vaccine strategy 559 (Vargas et al. 2015, Colombo et al. 2019, Freitas et al. 2019, Vargas et al. 2020). However, to date cryptococcal EVs have not been tested in murine infection models.

In a pilot experiment, we obtained EVs from C. neoformans wild-type strain and the acapsular cap59 mutant, used them to immunize BALB/c mice in two different EV-protein dosages (1 and $10 \mu \mathrm{g})$ via intraperitoneal injections; control group was injected with only PBS

564 (four mice in each group). After three immunizations, anti-EV-antibody response was 
565 evaluated in the mouse sera. Regardless of the EV origin, all the immunized mice produced 566 antibodies against vesicular proteins, as revealed by Western Blot (Fig. 10A). Forty days after 567 the last immunization, mice were challenged intranasally with $C$. neoformans wild type strain 568 ( 1 x $10^{4}$ yeasts per mouse), and their survival were monitored post-infection. All EV569 immunized mice survived longer than the non-immunized ones and immunization with both 570 doses of cap59 $\mathrm{EVs}$ statistically significantly prolonged the survival of the mice (Fig. 10B) 571 To note, the total carbohydrate per $100 \mu \mathrm{g}$ of EV-proteins were approximately $22 \mu \mathrm{g}$ and 3 $572 \mu \mathrm{g}$, respectively, for wild-type and cap59 mutant, as analyzed by gas-chromatography 573 analyses (Richie et al. 2009). We then confirmed this result using a larger number of mice (10 574 mice per group). Since the highest dose of EVs from the acapsular mutant rendered the best 575 protection, we decided to proceed only with EVs from cap59 strain (10 $\mu \mathrm{g}$ per mouse). After 576 immunizations with EVs, the anti-EV-antibody response in the mice was analyzed; all 577 immunized mice produced antibodies against vesicular molecules (Fig. 10C). Following, the 578 mice were challenged with $C$. neoformans wild type strain ( $1 \times 10^{4}$ yeasts per mouse), and 579 their survival was monitored post-infection. EV-immunization led to a significant prolonged 580 survival ( $p=0.0006)$ (Fig. 10D), thus confirming the promising potential usage of EV-based protection against Cryptococcus.

\section{Discussion}

Studies on fungal EVs have gained much attention during recent years (Rizzo et al. 2020).

585 Although data from both pathogenic and nonpathogenic species highlight their importance in 586 diverse biological contexts, knowledge on fungal EVs is still limited, mostly due to their nanometer size and the technical hurdles intrinsic to the methods applied for their characterization (Rizzo et al. 2020). Here we used cutting edge technologies to revisit Cryptococcus EVs. Our cryo-EM analysis produced an unprecedented quality of EV images and resolved the fibrillar structure decoration as a new aspect on fungal EVs.

Our hypothesis is that EV decoration is not capsular polysaccharide GXM-based but mainly composed of mannoproteins. This is supported by two independent experiments. First,

593 we demonstrated that although GXM most probably surrounds the vesicles, it is not necessary 594 for the presence of decoration. Thus, EVs produced by an acapsular strain of $C$. neoformans are not bound by a GXM specific antibody yet still display decoration. Secondly, C. albicans and S. cerevisiae EVs are also decorated, although none of these yeasts produced a capsular 
598

599

600

601

602

603

604

605

606

607

608

609

610

611

612

613

614

615

616

617

618

619

620

621

622

623

624

625

626

627

628

629

630

such as the GPI-anchored proteins Mp88 and Vep1, was not sufficient to completely remove the EV decoration, suggesting that this structure has a highly complex and dynamic composition, including several mannoproteins.

Indeed, previous reports in C. albicans showed that the role of GPI-anchored proteins are redundant and single mutants mostly displayed minor phenotypes, if any (Plaine et al. 2008). Interestingly, Johansson and coworkers performed cryo-EM analysis of Malassezia sympodialis EVs, demonstrating no (Johansson et al. 2018) evident decoration on their surfaces (Johansson et al. 2018). Comparative genomic analysis suggested that this lipophilic pathogenic yeast, living on the skin (Theelen et al. 2018), lacks the N-glycosylation pathway and possesses only a very small number of GPI-anchor proteins (Gioti et al. 2013). Accordingly, M. sympodialis cells lack the extensive mannan outer fibrillar layer, which can be easily observed at the surface of the cell wall of most yeasts including $S$. cerevisiae or $C$. albicans (Gioti et al. 2013, Muszewska et al. 2017). Therefore, it is very tempting to hypothesize that this absence of mannans in M. sympodialis could explain the absence of EV decoration, supporting the idea that EV decoration in Cryptococcus species is mannoproteinbased. Previous proteomic analysis of fungal EVs identified putative mannoproteins, suggesting that this decoration is a common feature of fungal EVs (Bleackley et al. 2019, Dawson et al. 2020, Karkowska-Kuleta et al. 2020, Rizzo et al. 2020). Accordingly, flow cytometry experiments showed that $C$. glabrata EVs can be labelled by ConA (KarkowskaKuleta et al. 2020). Putative fibril-like structures have also been reported at the surface of Aspergillus fumigatus EVs produced during cell wall regeneration (Rizzo et al. 2020).

In addition, we performed proteome analysis of EVs from S. cerevisiae and $C$. albicans grown in the same conditions as Cryptococcus species, and confirmed the presence of number of cell wall and GPI-anchored proteins in their EVs (Vargas et al. 2015, Zhao et al. 2019, Dawson et al. 2020). We also confirmed the presence of diverse antigenic proteins associated with EVs in C. albicans, reinforcing the notion that this feature might be a general aspect of pathogenic fungal EVs (Table S5). Whereas the presence of decoration seems to be a hallmark of fungal EV, it is not specific to this kingdom (Macedo-da-Silva et al. 2021). Although EVs bearing visible structures on their surface have not been commonly reported, a recent cryo-EM analysis of EVs derived from human breast cell lines overexpressing hyaluronan synthase 3-(HAS3) suggested the presence of fibril-like structures on their vesicle surface (Noble et al. 2020). Additionally, EVs from poliovirus-infected cells contain 'protein structures with globular heads on a stalk' around the membrane (Yang et al. 2020). 
631 Nevertheless, it is still unclear how often this feature is present among the whole EV 632 population, and what the composition of these surface structures is.

633 Previous studies explored the size and morphology of fungal EVs, mostly by 634 techniques such as electron microscopy (TEM, SEM), dynamic light scattering (DLS), and 635 NTA (Albuquerque et al. 2008, Rodrigues et al. 2008, Wolf et al. 2014, Vargas et al. 2015, 636 Wolf et al. 2015, Bielska and May 2019). Here we show that cryptococcal EVs are more 637 heterogeneous than previously recognized in terms of size distribution and morphotypes. Our 638 cryo-EM analysis revealed that the peak of EV size distribution was smaller than $100 \mathrm{~nm}$, and 639 substantially different from size distribution observed by NTA and from that previously found 640 from $C$. neoformans and C. deuterogattii EVs using NTA and DLS approaches (100 to 300 $641 \mathrm{~nm}$ ) (Reis et al. 2019). Moreover, our study revealed not only the presence of regular EVs but 642 also tubular, flat, and multilayered EVs. Although the different EV morphologies were 643 previously identified in many fungal pathogens (Albuquerque et al. 2008, Rodrigues et al. 644 2008, Tefsen et al. 2014, Vargas et al. 2015), some vesicular shapes found in this work have 645 not previously been reported. Thus, membrane tubule structures (memtubs) budding from the 646 plasma membrane were found in the arbuscular fungus Rhizophagus irregularis, suggesting 647 that different shapes of membranous structures could appear during fungal growth (Roth et al. 648 2019). Additionally, tubular and other morphologies were also found in EV populations 649 obtained from human biological fluids (Arraud et al. 2014, Emelyanov et al. 2020). Although 650 these data suggest that diverse structures could be part of the native EV population, the 651 cellular origins of these structures are still unknown, and we cannot rule out the possibility of them being artifacts resulted from the filtration step of the commonly used EV isolation protocols.

654 In this study, we demonstrated that the three Cryptococcus species release both 655 decorated and undecorated EVs, adding another previously unappreciated aspect to fungal EV 656 diversity. As hypothesized before, this result also suggests the existence of at least two 657 different pathways involved in EV biogenesis (Oliveira et al. 2010, Oliveira et al. 2013, 658 Bielska and May 2019, Rizzo et al. 2020). It is, therefore, reasonable to hypothesize that 659 decorated EVs could be shed from the fungal plasma membrane, "stealing" cell membrane 660 proteins when they bud out. Interestingly, the decorated EVs have larger size distribution than 661 the undecorated ones, in good agreement with what would be typical microvesicles in 662 mammals. In this hypothesis, the enrichment of tetraspanin membrane proteins containing a 663 SUR7/PalI family motif might indicate that decorated EVs could be specifically shed from the 664 Sur7 specialized plasma membrane domains. This model could be extended to other fungi as 
665 Sur7 proteins have been recently identified as EV-protein markers in C. albicans and in the 666 wheat pathogen Zymoseptoria tritici (Dawson et al. 2020, Hill and Solomon 2020). This latter hypothesis, together with whether or not the smaller undecorated EVs are a result of the endosomal secretory pathways, thought to be exosomes being released by multivesicular bodies (MVB), still needs to be further explored. Interestingly, the characterization of decorated and undecorated EVs as microvesicles and exosomes, respectively, has previously been proposed (Noble et al. 2020). This hypothesis and our current results are supported by a recent study of A. fumigatus EVs in the absence of a cell wall. EVs were formed at the plasma membrane level and they contained a number of plasma membrane proteins (Rizzo et al. 674 2020).

Our work suggests that cryptococcal EV cargo contains proteins involved in diverse biological processes, including Mp88 and members of Cda and Gox families, which have been suggested as immunomodulators (Specht et al. 2017, Hester et al. 2020). Since the novel surface structure on fungal EVs resolved by cryo-EM resembles the spike complexes on viral envelopes (Neuman et al. 2006, Zanetti et al. 2006), we reasoned they may be useful as a vaccine platform. Numerous efforts are underway to develop vaccines against fungal infections, although none have yet been approved for human use (Nami et al. 2019). It was previously shown that the pre-treatment of Galleria mellonella larvae with fungal EVs stimulated a protective response against a lethal challenge with C. albicans, C. neoformans or A. fumigatus (Vargas et al. 2015, Colombo et al. 2019, Brauer et al. 2020). More recently, it was also demonstrated that $C$. albicans EVs were also able to elicit a protective effect against murine candidiasis (Vargas et al. 2020). Interestingly, C. neoformans EVs show immunoreactivity with sera from patients with cryptococcosis, indicating that EV-associated proteins are produced during cryptococcal infection (Rodrigues et al. 2008). Prophylactic immunization is one of the effective methods to prevent cryptococcal infection, and several cryptococcal antigens have been tested for their vaccination potential (Caballero Van Dyke and Wormley 2018, Ueno et al. 2020). However, the in vivo immunoregulatory role of EVs have largely remained unknown (Robbins and Morelli 2014).

In our study, antibody responses in cryptococcal EV-immunized mice indicate that the EVs can elicit an adaptive immune response in the absence of any adjuvants or carriers, unlike other antigenic proteins of Cryptococcus (Specht et al. 2017). It is also important to note that immunization using $C$. neoformans heat-killed cells does not elicit protection in a murine model of infection (Masso-Silva et al. 2018). EV-based vaccination data obtained by 
699 (Vargas et al. 2015, Colombo et al. 2019). As Cryptococcus predominantly infects 700 immunocompromised hosts, it will be worth checking the role of EVs in eliciting trained 701 immunity, wherein innate immune cells develop memory-like response against an antigen 702 upon repeated exposure (Hole et al. 2019, Mulder et al. 2019). The mechanisms, and the 703 responsible immune cell types leading to prolonged survival in our murine infection model, 704 remain to be deciphered. Although EV immunization was not sufficient to prevent death, we 705 believe that adjusting the antigens exposed on EV surface could potentially increase the 706 protective effect. In that sense, the fact that EVs from C. neoformans WT and the acapsular 707 mutant did not lead to the same level of protection is an encouraging data.

708 Overall, the fantastic power of cryo-EM, together with several innovative analyses, 709 has enabled us to draw a new model of fungal EVs and revealed new aspects of their 710 diversity, suggesting different biosynthetic pathways. This model supports new strategies to 711 construct vaccines against these too often neglected infectious diseases. It also opens the door 712 to more questions concerning the origin and the fate of fungal EVs. 


\section{References}

Albuquerque PC, Nakayasu ES, Rodrigues ML, Frases S, Casadevall A, Zancope-Oliveira RM, Almeida IC, Nosanchuk JD 2008. Vesicular transport in Histoplasma capsulatum: an effective mechanism for trans-cell wall transfer of proteins and lipids in ascomycetes. Cell Microbiol 10: 1695-1710. Almagro Armenteros JJ, Tsirigos KD, Sønderby CK, Petersen TN, Winther O, Brunak S, von Heijne G, Nielsen H 2019. SignalP 5.0 improves signal peptide predictions using deep neural networks. Nature Biotechnol 37: 420-423.

Arraud N, Linares R, Tan S, Gounou C, Pasquet JM, Mornet S, Brisson AR 2014. Extracellular vesicles from blood plasma: determination of their morphology, size, phenotype and concentration. Journal of Thrombosis and Haemostasis 12: 614-627.

Bachurski D, Schuldner M, Nguyen P-H, Malz A, Reiners KS, Grenzi PC, Babatz F, Schauss AC, Hansen $\mathrm{HP}$, et al. 2019. Extracellular vesicle measurements with nanoparticle tracking analysis - An accuracy and repeatability comparison between NanoSight NS300 and ZetaView. J Extracell Vesicles 8: 1596016-1596016.

Baker LG, Specht CA, Lodge JK 2011. Cell Wall Chitosan Is Necessary for Virulence in the Opportunistic Pathogen Cryptococcus neoformans. Eukaryot Cell 10: 1264.

Bielska E, May RC 2019. Extracellular vesicles of human pathogenic fungi. Curr Opinion Microbiol 52: 90-99.

Bielska E, Sisquella MA, Aldeieg M, Birch C, O'Donoghue EJ, May RC 2018. Pathogen-derived extracellular vesicles mediate virulence in the fatal human pathogen Cryptococcus gattii. Nature Com 9: 1556-1556.

Bleackley MR, Dawson CS, Anderson MA 2019. Fungal extracellular vesicles with a focus on proteomic analysis. PROTEOMICS 19: 1800232.

Bleackley MR, Samuel M, Garcia-Ceron D, McKenna JA, Lowe RGT, Pathan M, Zhao K, Ang C-S, Mathivanan S, et al. 2020. Extracellular Vesicles From the Cotton Pathogen Fusarium oxysporum $\mathrm{f}$. sp. vasinfectum Induce a Phytotoxic Response in Plants. Frontiers in plant science 10: 1610-1610.

Brauer VS, Pessoni AM, Bitencourt TA, de Paula RG, de Oliveira Rocha L, Goldman GH, Almeida F 2020. Extracellular vesicles from Aspergillus flavus induce M1 polarization in vitro. mSphere 5 : e00190-00120.

Caballero Van Dyke MC, Wormley FL, Jr. 2018. A Call to Arms: Quest for a Cryptococcal Vaccine. Trends Microbiol 26: 436-446.

Casadevall A, Mukherjee J, Devi SJN, Schneerson R, Robbins JB, Scharff MD 1992. Antibodies elicited by a Cryptococcus neoformans-tetanus toxoid conjugate vaccine have the same specificity as those elicited in infection. The Journal of Infectious Diseases 165: 1086-1093.

Chiang C-Y, Chen C 2019. Toward characterizing extracellular vesicles at a single-particle level. J Biomed Sci 26: 9-9.

Chiou N-T, Kageyama R, Ansel KM 2018. Selective Export into Extracellular Vesicles and Function of tRNA Fragments during T Cell Activation. Cell Rep. 25: 3356-3370.e3354.

Chun CD, Brown JCS, Madhani HD 2011. A major role for capsule-independent phagocytosisinhibitory mechanisms in mammalian infection by Cryptococcus neoformans. Cell host \& microbe 9: 243-251.

Coakley G, McCaskill JL, Borger JG, Simbari F, Robertson E, Millar M, Harcus Y, McSorley HJ, Maizels RM, et al. 2017. Extracellular Vesicles from a Helminth Parasite Suppress Macrophage Activation and Constitute an Effective Vaccine for Protective Immunity. Cell Rep. 19: 1545-1557. construction of gene deletion cassettes for generation of Neurospora crassa knockout strains. 
Colombo AC, Rella A, Normile T, Joffe LS, Tavares PM, de S. Araújo GR, Frases S, Orner EP, Farnoud AM, et al. 2019. Cryptococcus neoformans glucuronoxylomannan and sterylglucoside are required for host protection in an animal vaccination model. mBio 10: e02909-02918.

Cox J, Mann M 2008. MaxQuant enables high peptide identification rates, individualized p.p.b.-range mass accuracies and proteome-wide protein quantification. Nature Biotechnol 26: 1367-1372.

Cox J, Neuhauser N, Michalski A, Scheltema RA, Olsen JV, Mann M 2011. Andromeda: A Peptide Search Engine Integrated into the MaxQuant Environment. Journal of Proteome Research 10: 17941805.

da Silva RP, Puccia R, Rodrigues ML, Oliveira DL, Joffe LS, César GV, Nimrichter L, Goldenberg S, Alves LR 2015. Extracellular vesicle-mediated export of fungal RNA. Sci Rep 5: 7763.

Dawson CS, Garcia-Ceron D, Rajapaksha H, Faou P, Bleackley MR, Anderson MA 2020. Protein markers for Candida albicans EVs include claudin-like Sur7 family proteins. I Extracell Vesicles 9: 1750810.

de Groot PWJ, Hellingwerf KJ, Klis FM 2003. Genome-wide identification of fungal GPI proteins. Yeast 20: 781-796

Deatherage BL, Cookson BT 2012. Membrane Vesicle Release in Bacteria, Eukaryotes, and Archaea: a Conserved yet Underappreciated Aspect of Microbial Life. Infect Immun 80: 1948.

Eisenman HC, Frases S, Nicola AM, Rodrigues ML, Casadevall A 2009. Vesicle-associated melanization in Cryptococcus ne oformans. Microbiology 155: 3860-3867.

Emelyanov A, Shtam T, Kamyshinsky R, Garaeva L, Verlov N, Miliukhina I, Kudrevatykh A, Gavrilov G, Zabrodskaya $Y$, et al. 2020. Cryo-electron microscopy of extracellular vesicles from cerebrospinal fluid. PLOS ONE 15: e0227949.

Fan Y, Lin X 2018. Multiple Applications of a Transient CRISPR-Cas9 Coupled with Electroporation (TRACE) System in the Cryptococcus neoformans Species Complex. Genetics 208: 1357.

Freitas MS, Bonato VLD, Pessoni AM, Rodrigues ML, Casadevall A, Almeida F 2019. Fungal Extracellular Vesicles as Potential Targets for Immune Interventions. mSphere 4: e00747-00719.

Garcia-Santamarina S, Probst C, Festa RA, Ding C, Smith AD, Conklin SE, Brander S, Kinch LN, Grishin NV, et al. 2020. A lytic polysaccharide monooxygenase-like protein functions in fungal copper import and meningitis. Nat Chem Biol 16: 337-344.

Gioti A, Nystedt B, Li W, Xu J, Andersson A, Averette AF, Münch K, Wang X, Kappauf C, et al. 2013. Genomic Insights into the Atopic Eczema-Associated Skin Commensal Yeast \&lt;em\&gt;Malassezia sympodialis\&lt;/em\&gt. mBio 4: e00572-00512.

Gonzalez-Hilarion S, Paulet D, Lee K-T, Hon C-C, Lechat P, Mogensen E, Moyrand F, Proux C, Barboux $\mathrm{R}$, et al. 2016. Intron retention-dependent gene regulation in Cryptococcus neoformans. Sci Rep 6: 32252.

Gröhs Ferrareze PA, Maufrais C, Silva Araujo Streit R, Priest SJ, Cuomo CA, Heitman J, Staats CC, Janbon $G$ 2021. Application of an optimized annotation pipeline to the Cryptococcus deuterogattii genome reveals dynamic primary metabolic gene clusters and genomic impact of RNAi loss. G3 (Bethesda) 11.

Hagen F, Khayhan K, Theelen B, Kolecka A, Polacheck I, Sionov E, Falk R, Parnmen S, Lumbsch HT, et al. 2015. Recognition of seven species in the Cryptococcus gattii/Cryptococcus neoformans species complex. Fungal Genet Biol 78: 16-48.

Hai TP, Tuan TL, Van Anh D, Mai TN, Phu Huong LN, Thwaites GE, Johnson E, Van Vinh Chau N, Ashton $P$, et al. 2020. The expression of virulence by the Cryptococcus neoformans VNla-5 lineage is plastic and associated with host immune background. bioRxiv: 2020.2002.2024.962134.

Hester MM, Lee CK, Abraham A, Khoshkenar P, Ostroff GR, Levitz SM, Specht CA 2020. Protection of mice against experimental cryptococcosis using glucan particle-based vaccines containing novel recombinant antigens. Vaccine 38: 620-626.

Hill E, Solomon P (2020). Extracellular Vesicles from the Apoplastic Fungal Wheat Pathogen Zymoseptoria Tritici, Research Square.

Hole CR, Wager CML, Castro-Lopez N, Campuzano A, Cai H, Wozniak KL, Wang Y, Wormley FL 2019. Induction of memory-like dendritic cell responses in vivo. Nature Com 10: 2955. 
815 Huang C, Nong SH, Mansour MK, Specht CA, Levitz SM 2002. Purification and characterization of a 816 second immunoreactive mannoprotein from Cryptococcus neoformans that stimulates T-cell 817 responses. Infect Immun 70: 5485-5493.

818 Janbon G, Ormerod KL, Paulet D, Byrnes III EJ, Chatterjee G, Yadav V, Mullapudi N, Hon CC, Billmyre 819 RB, et al. 2014. Analysis of the genome and transcriptome of Cryptococcus neoformans var. grubii 820 reveals complex RNA expression and microevolution leading to virulence attenuation. PLoS Genet 10: 821 e1004261.

822 Janbon G, Quintin J, Lanternier F, d'Enfert C 2019. Studying fungal pathogens of humans and fungal 823 infections: fungal diversity and diversity of approaches. Genes Immun 20: 403-414. Joffe LS, Nimrichter L, Rodrigues ML, Del Poeta M 2016. Potential Roles of Fungal Extracellular Vesicles during Infection. mSphere 1: e00099-00016.

Johansson HJ, Vallhov H, Holm T, Gehrmann U, Andersson A, Johansson C, Blom H, Carroni M, Lehtiö $\mathrm{J}$, et al. 2018. Extracellular nanovesicles released from the commensal yeast Malassezia sympodialis are enriched in allergens and interact with cells in human skin. Sci Rep 8: 9182.

Jung WH, Sham A, Lian T, Singh A, Kosman DJ, Kronstad JW 2008. Iron source preference and regulation of iron uptake in Cryptococcus neoformans. PLoS Pathog 4: e45.

Karkowska-Kuleta J, Kulig K, Karnas E, Zuba-Surma E, Woznicka O, Pyza E, Kuleta P, Osyczka A, RapalaKozik M, et al. 2020. Characteristics of extracellular vesicles released by the pathogenic yeast-like fungi Candida glabrata, Candida parapsilosis and Candida tropicalis. Cells 9: 1722.

Kremer JR, Mastronarde DN, McIntosh JR 1996. Computer Visualization of Three-Dimensional Image Data Using IMOD. Journal of Structural Biology 116: 71-76.

Krogh A, Larsson B, von Heijne G, Sonnhammer ELL 2001. Predicting transmembrane protein topology with a hidden markov model: application to complete genomes. J Mol Biol 305: 567-580.

Kwon-Chung KJ, Fraser JA, Doering TL, Wang Z, Janbon G, Idnurm A, Bahn YS 2014. Cryptococcus neoformans and Cryptococcus gattii, the etiologic agents of cryptococcosis. Cold Spring Harb Perspect Med 4: a019760.

Lee DJ, Bahn YS, Kim HJ, Chung SY, Kang HA 2015. Unraveling the novel structure and biosynthetic pathway of O-linked glycans in the Golgi apparatus of the human pathogenic yeast Cryptococcus neoformans. J Biol Chem 290: 1861-1873.

Leone F, Bellani L, Muccifora S, Giorgetti L, Bongioanni P, Simili M, Maserti B, Del Carratore R 2018. Analysis of extracellular vesicles produced in the biofilm by the dimorphic yeast Pichia fermentans. Journal of Cellular Physiology 233: 2759-2767.

Levitz SM, Nong S, Mansour MK, Huang C, Specht CA 2001. Molecular characterization of a mannoprotein with homology to chitin deacetylases that stimulates T cell responses to Cryptococcus neoformans. Proc Natl Acad Sci USA 98: 10422-10427.

Maas SLN, de Vrij J, van der Vlist EJ, Geragousian B, van Bloois L, Mastrobattista E, Schiffelers RM, Wauben MHM, Broekman MLD, et al. 2015. Possibilities and limitations of current technologies for quantification of biological extracellular vesicles and synthetic mimics. Journal of Controlled Release 200: 87-96.

Macedo-da-Silva J, Santiago VF, Rosa-Fernandes L, Marinho CRF, Palmisano G 2021. Protein glycosylation in extracellular vesicles: Structural characterization and biological functions. $\mathrm{Mol}$ Immunol 135: 226-246.

Masso-Silva J, Espinosa V, Liu T-B, Wang Y, Xue C, Rivera A 2018. The F-Box protein Fbp1 shapes the immunogenic potential of Cryptococcus neoformans. mBio 9: e01828-01817.

Mastronarde DN 2005. Automated electron microscope tomography using robust prediction of specimen movements. Journal of Structural Biology 152: 36-51.

Mastronarde DN, Held SR 2017. Automated tilt series alignment and tomographic reconstruction in IMOD. Journal of structural biology 197: 102-113.

Meldolesi J 2018. Exosomes and Ectosomes in Intercellular Communication. Curr Biol 28: R435-R444. Moyrand F, Fontaine T, Janbon G 2007. Systematic capsule gene disruption reveals the central role of galactose metabolism on Cryptococcus neoformans virulence. Mol Microbiol 64: 771-781. 
866 Mulder WJM, Ochando J, Joosten LAB, Fayad ZA, Netea MG 2019. Therapeutic targeting of trained 867 immunity. Nat Rev Drug Discov 18: 553-566.

868 Muszewska A, Piłsyk S, Perlińska-Lenart U, Kruszewska JS 2017. Diversity of Cell Wall Related Proteins 869 in Human Pathogenic Fungi. Journal of fungi (Basel, Switzerland) 4: 6.

870 Nami S, Mohammadi R, Vakili M, Khezripour K, Mirzaei H, Morovati H 2019. Fungal vaccines, 871 mechanism of actions and immunology: A comprehensive review. Biomedicine \& Pharmacotherapy 872 109: 333-344.

873 Neuman BW, Adair BD, Yoshioka C, Quispe JD, Orca G, Kuhn P, Milligan RA, Yeager M, Buchmeier MJ 874 2006. Supramolecular architecture of severe acute respiratory syndrome coronavirus revealed by 875 electron cryomicroscopy. J Virol 80: 7918-7928.

876 Noble JM, Roberts LM, Vidavsky N, Chiou AE, Fischbach C, Paszek MJ, Estroff LA, Kourkoutis LF 2020. 877 Direct comparison of optical and electron microscopy methods for structural characterization of 878 extracellular vesicles. Journal of Structural Biology 210: 107474.

879 O'Meara TR, Alspaugh JA 2012. The Cryptococcus neoformans capsule: a sword and a shield. Clin 880 Microbiol Rev 25: 387-408.

881 Oliveira DL, Freire-de-Lima CG, Nosanchuk JD, Casadevall A, Rodrigues ML, Nimrichter L 2010. 882 Extracellular vesicles from Cryptococcus neoformans modulate macrophage functions. Infect Immun 883 78: 1601-1609.

884 Oliveira DL, Nakayasu ES, Joffe LS, Guimarães AJ, Sobreira TJP, Nosanchuk JD, Cordero RJB, Frases S, 885 Casadevall A, et al. 2010. Characterization of Yeast Extracellular Vesicles: Evidence for the Participation of Different Pathways of Cellular Traffic in Vesicle Biogenesis. PLOS ONE 5: e11113. Oliveira DL, Nimrichter L, Miranda K, Frases S, Faull KF, Casadevall A, Rodrigues ML 2009. Cryptococcus neoformans cryoultramicrotomy and vesicle fractionation reveals an intimate association between membrane lipids and glucuronoxylomannan. Fungal Genet Biol 46: 956-963.

Oliveira DL, Rizzo J, Joffe LS, Godinho RMC, Rodrigues ML 2013. Where do they come from and where do they go: candidates for regulating extracellular vesicle formation in fungi. Int J Mol Sci 14: 9581-9603.

Orlov I, Myasnikov AG, Andronov L, Natchiar SK, Khatter H, Beinsteiner B, Ménétret J-F, Hazemann I, Mohideen K, et al. 2017. The integrative role of cryo electron microscopy in molecular and cellular structural biology. Biology of the Cell 109: 81-93.

Pettersen EF, Goddard TD, Huang CC, Couch GS, Greenblatt DM, Meng EC, Ferrin TE 2004. UCSF Chimera-A visualization system for exploratory research and analysis. Journal of Computational Chemistry 25: 1605-1612.

Pierleoni A, Martelli PL, Casadio R 2008. PredGPI: a GPI-anchor predictor. BMC Bioinformatics 9: 392. Plaine A, Walker L, Da Costa G, Mora-Montes HM, McKinnon A, Gow NAR, Gaillardin C, Munro CA, Richard ML 2008. Functional analysis of Candida albicans GPI-anchored proteins: roles in cell wall integrity and caspofungin sensitivity. Fungal genetics and biology : FG \& B 45: 1404-1414.

Rajasingham R, Smith RM, Park BJ, Jarvis JN, Govender NP, Chiller TM, Denning DW, Loyse A, Boulware DR 2017. Global burden of disease of HIV-associated cryptococcal meningitis: an updated analysis. The Lancet Infectious Diseases 17: 873-881.

Rayner S, Bruhn S, Vallhov H, Andersson A, Billmyre RB, Scheynius A 2017. Identification of small RNAs in extracellular vesicles from the commensal yeast Malassezia sympodialis. Sci Rep 7: 39742.

Reis FCG, Borges BS, Jozefowicz LJ, Sena BAG, Garcia AWA, Medeiros LC, Martins ST, Honorato L, Schrank A, et al. 2019. A Novel Protocol for the Isolation of Fungal Extracellular Vesicles Reveals the Participation of a Putative Scramblase in Polysaccharide Export and Capsule Construction in Cryptococcus gattii. mSphere 4: e00080-00019.

Reis FCG, Costa JH, Honorato L, Nimrichter L, Fill TP, Rodrigues ML 2021. Small Molecule Analysis of Extracellular Vesicles Produced by Cryptococcus gattii: Identification of a Tripeptide Controlling Cryptococcal Infection in an Invertebrate Host Model. Front Immunol 12.

Richie DL, Hartl L, Aimanianda V, Winters MS, Fuller KK, Miley MD, White S, McCarthy JW, Latgé J-P, et al. 2009. A role for the unfolded protein response (UPR) in virulence and antifungal susceptibility in Aspergillus fumigatus. PLoS Pathog 5: e1000258-e1000258. 
918 Rizzo J, Albuquerque PC, Wolf JM, Nascimento R, Pereira MD, Nosanchuk JD, Rodrigues ML 2017. 919 Analysis of multiple components involved in the interaction between Cryptococcus neoformans and 920 Acanthamoeba castellanii. Fungal Biology 121: 602-614.

921 Rizzo J, Chaze T, Miranda K, Roberson RW, Gorgette O, Nimrichter L, Matondo M, Latgé J-P, Beauvais 922 A, et al. 2020. Characterization of extracellular vesicles produced by Aspergillus fumigatus 923 protoplasts. mSphere 5: e00476-00420.

924 Rizzo J, Rodrigues ML, Janbon G 2020. Extracellular Vesicles in Fungi: Past, Present, and Future 925 Perspectives. Front Cell Infect Microbiol 10.

926 Robbins PD, Morelli AE 2014. Regulation of immune responses by extracellular vesicles. Nat Rev 927 Immunol 14: 195-208.

928 Rodrigues ML, Casadevall A 2018. A two-way road: novel roles for fungal extracellular vesicles. Mol 929 Microbiol 110: 11-15.

930 Rodrigues ML, Godinho RMC, Zamith-Miranda D, Nimrichter L 2015. Traveling into Outer Space: 931 Unanswered Questions about Fungal Extracellular Vesicles. PLoS Pathog 11: e1005240.

932 Rodrigues ML, Nakayasu ES, Oliveira DL, Nimrichter L, Nosanchuk JD, Almeida IC, A. C 2008. 933 Extracellular vesicles produced by Cryptococcus neoformans contain protein components associated 934 with virulence. Eukaryot Cell 7: 58-67.

935 Rodrigues ML, Nimrichter L, Oliveira DL, Frases S, Miranda K, Zaragoza O, Alvarez M, Nakouzi A, 936 Feldmesser $\mathrm{M}$, et al. 2007. Vesicular polysaccharide export in Cryptococcus neoformans is a

eukaryotic solution to the problem of fungal trans-cell wall transport. Eukaryot Cell 6: 48-59.

Roth R, Hillmer S, Funaya C, Chiapello M, Schumacher K, Lo Presti L, Kahmann R, Paszkowski U 2019. Arbuscular cell invasion coincides with extracellular vesicles and membrane tubules. Nature Plants 5 : 204-211.

Schorb M, Haberbosch I, Hagen WJH, Schwab Y, Mastronarde DN 2019. Software tools for automated transmission electron microscopy. Nature Meth 16: 471-477.

Schwanhäusser B, Busse D, Li N, Dittmar G, Schuchhardt J, Wolf J, Chen W, Selbach M 2011. Global quantification of mammalian gene expression control. Nature 473: 337-342.

Shopova IA, Belyaev I, Dasari P, Jahreis S, Stroe MC, Cseresnyés Z, Zimmermann A-K, Medyukhina A, Svensson C-M, et al. 2020. Human Neutrophils Produce Antifungal Extracellular Vesicles against Aspergillus fumigatus. mBio 11: e00596-00520.

Souza JAM, Baltazar LdM, Carregal VM, Gouveia-Eufrasio L, de Oliveira AG, Dias WG, Campos Rocha M, Rocha de Miranda K, Malavazi I, et al. 2019. Characterization of Aspergillus fumigatus extracellular vesicles and their effects on macrophages and neutrophils functions. Front Microbiol 10: 2008-2008.

Specht CA, Lee CK, Huang H, Hester MM, Liu J, Luckie BA, Torres Santana MA, Mirza Z, Khoshkenar P, et al. 2017. Vaccination with Recombinant Cryptococcus Proteins in Glucan Particles Protects Mice against Cryptococcosis in a Manner Dependent upon Mouse Strain and Cryptococcal Species. mBio 8: e01872-01817.

Tefsen B, Grijpstra J, Ordonez S, Lammers M, van Die I, de Cock H 2014. Deletion of the CAP10 gene of Cryptococcus neoformans results in a pleiotropic phenotype with changes in expression of virulence factors. Research in Microbiology 165: 399-410.

Thak EJ, Lee S-B, Xu-Vanpala S, Lee D-J, Chung S-Y, Bahn Y-S, Oh D-B, Shinohara ML, Kang HA 2020. Core $\mathrm{N}$-glycan structures are critical for the pathogenicity of Cryptococcus neoformans by modulating host cell death. mBio 11: e00711-00720.

Theelen B, Cafarchia C, Gaitanis G, Bassukas ID, Boekhout T, Dawson TL, Jr. 2018. Malassezia ecology, pathophysiology, and treatment. Med Mycol 56: S10-S25.

Théry C, Witwer KW, Aikawa E, Alcaraz MJ, Anderson JD, Andriantsitohaina R, Antoniou A, Arab T, Archer F, et al. 2018. Minimal information for studies of extracellular vesicles 2018 (MISEV2018): a position statement of the International Society for Extracellular Vesicles and update of the MISEV2014 guidelines. J Extracell Vesicles 7: 1535750-1535750.

Tyanova S, Temu T, Cox J 2016. The MaxQuant computational platform for mass spectrometry-based shotgun proteomics. Nature Protocols 11: 2301-2319. 
970 Ueno K, Yanagihara N, Shimizu K, Miyazaki Y 2020. Vaccines and protective immune memory 971 against Cryptococcosis. Biol. Pharm. Bull. 43.

972 Vallejo MC, Nakayasu ES, Longo LVG, Ganiko L, Lopes FG, Matsuo AL, Almeida IC, Puccia R 2012. 973 Lipidomic analysis of extracellular vesicles from the pathogenic phase of Paracoccidioides brasiliensis. 974 Plos one 7: e39463-e39463.

975 Van Der Pol E, Hoekstra AG, Sturk A, Otto C, Van Leeuwen TG, Nieuwland R 2010. Optical and non976 optical methods for detection and characterization of microparticles and exosomes. Journal of 977 Thrombosis and Haemostasis 8: 2596-2607.

978 van Niel G, D'Angelo G, Raposo G 2018. Shedding light on the cell biology of extracellular vesicles. 979 Nature Reviews Molecular Cell Biology 19: 213-228.

980 Vargas G, Honorato L, Guimarães AJ, Rodrigues ML, Reis FCG, Vale AM, Ray A, Nosanchuk JD, 981 Nimrichter $L$ 2020. Protective effect of fungal extracellular vesicles against murine candidiasis. Cell 982 Microbiol n/a: e13238.

983 Vargas G, Rocha JDB, Oliveira DL, Albuquerque PC, Frases S, Santos SS, Nosanchuk JD, Gomes AMO, 984 Medeiros LCAS, et al. 2015. Compositional and immunobiological analyses of extracellular vesicles released by Candida albicans. Cell Microbiol 17: 389-407.

Wallace EWJ, Maufrais C, Sales-Lee J, Tuck LR, de Oliveira L, Feuerbach F, Moyrand F, Natarajan P, Madhani HD, et al. 2020. Quantitative global studies reveal differential translational control by start codon context across the fungal kingdom. Nucleic Acids Res 48: 2312-2331.

Wang X, Thompson CD, Weidenmaier C, Lee JC 2018. Release of Staphylococcus aureus extracellular vesicles and their application as a vaccine platform. Nature Com 9: 1379-1379.

Witwer KW, Théry C 2019. Extracellular vesicles or exosomes? On primacy, precision, and popularity influencing a choice of nomenclature. J Extracell Vesicles 8: 1648167.

Wolf JM, Espadas-Moreno J, Luque-Garcia JL, Casadevall A 2014. Interaction of Cryptococcus neoformans extracellular vesicles with the cell wall. Eukaryot Cell 13: 1484-1493.

Wolf JM, Espadas J, Luque-Garcia J, Reynolds T, Casadevall A 2015. Lipid biosynthetic genes affect Candida albicans extracellular vesicle morphology, cargo, and immunostimulatory properties. Eukaryot Cell 14: 745-754.

Xu R, Rai A, Chen M, Suwakulsiri W, Greening DW, Simpson RJ 2018. Extracellular vesicles in cancer - implications for future improvements in cancer care. Nature Reviews Clinical Oncology 15: 617638.

Yang B, Wang J, Jiang H, Lin H, Ou Z, Ullah A, Hua Y, Chen J, Lin X, et al. 2021. Extracellular vesicles derived from Talaromyces marneffei yeasts mediate inflammatory response in macrophage cells by bioactive protein components. Front Microbiol 11.

Yang JE, Rossignol ED, Chang D, Zaia J, Forrester I, Raja K, Winbigler H, Nicastro D, Jackson WT, et al. 2020. Complexity and ultrastructure of infectious extracellular vesicles from cells infected by nonenveloped virus. Sci Rep 10: 7939.

Zabeo D, Cvjetkovic A, Lässer C, Schorb M, Lötvall J, Höög JL 2017. Exosomes purified from a single cell type have diverse morphology. J Extracell Vesicles 6: 1329476-1329476.

Zanetti G, Briggs JAG, Grünewald K, Sattentau QJ, Fuller SD 2006. Cryo-Electron Tomographic Structure of an Immunodeficiency Virus Envelope Complex In Situ. PLoS Pathog 2: e83.

Zarnowski R, Sanchez H, Covelli AS, Dominguez E, Jaromin A, Bernhardt J, Mitchell KF, Heiss C, Azadi $P$, et al. 2018. Candida albicans biofilm-induced vesicles confer drug resistance through matrix biogenesis. PLoS Biol 16: e2006872.

Zhao K, Bleackley M, Chisanga D, Gangoda L, Fonseka P, Liem M, Kalra H, Al Saffar H, Keerthikumar S, et al. 2019. Extracellular vesicles secreted by Saccharomyces cerevisiae are involved in cell wall remodelling. Communications Biology 2: 305.

1017

1018

1019

\section{Geolocalisation}




$\begin{array}{llll}1020 & \text { Juliana RIZZO } & \text { ORCID } & 0000-0001-5538-6471 \\ 1021 & \text { Sarah Sze Wah WONG } & \text { ORCID } & 0000-0002-9440-1774 \\ 1022 & \text { Anastasia D. GAZI } & \text { ORCID } & 0000-0002-2922-3625 \\ 1023 & \text { Thibault CHAZE } & \text { ORCID } & 0000-0002-3615-7021 \\ 1024 & \text { Pierre-Henri COMMERE } & \text { ORCID } & 0000-0002-3886-4256 \\ 1025 & \text { Sophie NOVAULT } & \text { ORCID } & 0000-0001-5708-3597 \\ 1026 & \text { Mariette MATONDO } & \text { ORCID } & 0000-0003-3958-7710 \\ 1027 & \text { Gerard PEHAUD-ARNAUDET } & \text { ORCID } & 0000-0001-6479-9470 \\ 1028 & \text { Lysangela R ALVES } & \text { ORCID } & 0000-0002-1972-2658 \\ 1029 & \text { Robin C. MAY } & \text { ORCID } & 0000-0001-5364-1838 \\ 1030 & \text { Leonardo NIMRICHTER } & \text { ORCID } & 0000-0001-9281-6856 \\ 1031 & \text { Marcio L. RODRIGUES : } & \text { ORCID } & 0000-0002-6081-3439 \\ 1032 & \text { Vishukumar AIMANIANDA : } & \text { ORCID } & 0000-0001-5813-7497 \\ 1033 & \text { Guilhem JANBON : } & \text { ORCID } & 0000-0002-4788-1154\end{array}$

1034

1035

1036

1037

\section{Acknowledgements}

1038

1039

This work was supported by a CAPES COFECUB grant $n^{\circ} 39712 Z$ K (to GJ, MLR, LA and 1040 LN). JR was supported by the CAPES-COFECUB Franco-Brazilian Research Exchange 1041 Program (88887.357947/2019-00) and by a Pasteur-Roux-Cantarini fellowship of Institut 1042 Pasteur. SSWW was supported by CEFIPRA/ANR-DFG-AfuINF grant and Pasteur-Roux1043 Cantarini postdoctoral fellowship. VA was supported by ANR-DFG AfuINF and Indo-French 1044 Centre for the Promotion of Advanced Research (CEFIPRA; Grant N ${ }^{\circ}$ 5403-1) grants. Jean1045 Marie Winter from the NanoImaging Core at Institut Pasteur is acknowledged for his support 1046 image acquisition. The NanoImaging Core was created with the help of a grant from the 1047 French Government's 'Investissements d'Avenir' program (EQUIPEX CACSICE - "Centre 1048 d'analyse de systèmes complexes dans les environnements complexes", ANR-11-EQPX1049 0008). The Falcon II equipping the F20 microscope at the UBI used during this study was also 1050 financed by the Equipex CACSICE (grant number ANR-11-EQPX-0008). M.L.R. is 1051 supported by grants from the Brazilian Ministry of Health (grant 440015/2018-9), Conselho 1052 Nacional de Desenvolvimento Científico e Tecnológico (CNPq; grants 405520/2018-2 and 1053 301304/2017-3), and Fiocruz (grants VPPCB-007-FIO-18 and VPPIS-001-FIO18)MLR, JR, 1054 and FCGR also acknowledge support from Coordenac $\square$ ão de Aperfeic $\square$ oamento de Pessoal 1055 de Nível Superior (CAPES, finance code 001) and the Instituto Nacional de Cie $\square$ ncia e 1056 Tecnologia de Inovac $\square$ ão em Doenc $\square$ as de Populac $\square$ ões Negligenciadas (INCT-IDPN). 1057 MLR is currently on leave of an associate professor position at the Instituto de Microbiologia 
bioRxiv preprint doi: https://doi.org/10.1101/2020.08.17.253716; this version posted May 17, 2021. The copyright holder for this preprint (which

was not certified by peer review) is the author/funder, who has granted bioRxiv a license to display the preprint in perpetuity. It is made available under aCC-BY-NC-ND 4.0 International license.

1058 Paulo de Góes of the Universidade Federal do Rio de Janeiro. We thank Adèle Trottier for her 1059 help in the preparation of EV samples.

1060

1061 


\section{Figures legends}

1063

1064 Figure 1: Cryo-electron microscopy analysis of $C$. neoformans extracellular vesicles

1065 (EVs).

1066 Cryo-EM analysis revealed a heterogeneous population of vesicles with diverse structural 1067 aspects, previously unappreciated in fungal EVs (A). As shown, the EVs were delimitated by 1068 a lipid bilayer (B to E), which showed either no decoration (in 10.8\% vesicles, panels B and 1069 C) or a fibrillar decoration (arrows) in $89.2 \%$ of the EVs analyzed (panels D and E). Three1070 dimensional organization of the fibrillar decoration (yellow) on the membrane (purple) of 1071 EVs as revealed by cryo-electron tomography analysis $(\mathrm{F})$, magnified in panels $\mathrm{G}$ and H. Full 1072 surface representation models as seen from top view (G). Same models clipped with clipping 1073 plane oriented perpendicular to line of sight $(\mathrm{H})$. Data presented in this figure have been 1074 generated using images obtained using a Titan Krios (Thermo Scientific) transmission 1075 electron microscope.

1076

1077

Figure 2: Analysis of size and structural diversity of $C$. neoformans EVs.

1078

NTA analysis of purified EVs revealed a size diameter ranging from 80 to $500 \mathrm{~nm}$, with the 1079 highest distribution around $150 \mathrm{~nm}$ (A). Frequency distribution of EV diameters determined

1080 by CryoEM, a total of 434 regular EVs were analyzed. The analysis based on CryoEM

1081

tomograms revealed a wider range of $\mathrm{EV}$ size distribution, from 10 to $500 \mathrm{~nm}$ diameter, with

1082 the highest relative frequency below $100 \mathrm{~nm}$ (B). Cryo-EM images exemplifying EV size range. Scale bars corresponding to $100 \mathrm{~nm}(\mathrm{C})$. EV size distribution according to the presence or absence of surface decoration (D). Non-decorated EVs have a smaller diameter size distribution compared to decorated ones (E). Analysis of decoration thickness from Cryo-EM images from 105 single EVs (F). Analysis of a potential relationship between decoration thickness and EV diameter by linear regression $(\mathrm{G})$. Data presented in this figure have been generated using images obtained using a Titan Krios (Thermo Scientific) transmission electron microscope. Error bars show means \pm SD. Sample size (n) is indicated and, in

1090 brackets, the number of vesicles in that category that exceeded $500 \mathrm{~nm}$ in size.

Figure 3: Comparative analysis of size and structural diversity of EVs in C. neoformans, C. deneoformans and C. deuterogattii. Analysis of EV diameters revealed a smaller size distribution in C. deuterogattii strain R265 and $C$. deneoformans strain JEC21 than in $C$. neoformans KN99 $\alpha$. The total numbers of 
1096 vesicles analyzed were $C$. neoformans ( $\mathrm{n}=143$ for size and $\mathrm{n}=112$ for decoration), $C$. 1097 deneoformans ( $\mathrm{n}=90$ for size and $\mathrm{n}=63$ for decoration), C. deuterogattii $(\mathrm{n}=115$ for size and

1098

1099

1100

1101

1102

1103

1104

1105

1106

1107

1108

1109

1110

1111

1112

1113

1114

1115

1116

1117

1118

1119

1120

1121

1122

1123

1124

1125

1126

1127

1128

1129 $\mathrm{n}=95$ for decoration) (A). Analysis of the decoration thickness revealed a smaller distribution for $C$. deneoformans and $C$. deuterogattii compared with $C$. neoformans (B). Illustrative images of size and decoration of EVs obtained from the three species. The data presented in this figure have been generated using images obtained using a TECNAI F20 transmission electron microscope (C). Error bars show means \pm SD. Scale bars represent $100 \mathrm{~nm}$.

\section{Figure 4: Flow cytometry analysis of $C$. neoformans EVs incubated with monoclonal anti-GXM antibody.}

FACS analysis of wild type (WT) and the acapsular cap594 EVs in PBS or in the presence of the monoclonal antibody raised against the capsular polysaccharide $18 \mathrm{~b} 7$ (+ mAb anti-GXM) (A). The analysis revealed strong labelling of WT vesicles (74.7\%), compared to the weak labelling in the mutant (2.33\%), (B). Despite the important labelling difference, $C$. neoformans WT and cap594 strains released EVs bearing similar surface decoration, shown by the cryo-EM (arrows), as well as EVs obtained from other fungal species such as $C$. albicans and $S$. cerevisiae (C). These cryo-EM data have been generated using a TECNAI F20 transmission electron microscope. Scale bar represents $100 \mathrm{~nm}$. This experiment was repeated twice with similar results.

\section{Figure 5: Analysis of Cryptococcus spp protein cargo.}

Venn diagram revealing shared and unique EV-associated proteins in C. neoformans, $C$. deneoformans, and $C$. deuterogattii. Seventeen proteins were identified to be associated with EVs in all three Cryptococcus species (A). List of the gene loci and the corresponding proteins commonly found in EVs released by the three species, which could be considered as putative cryptococcal EV-protein markers (B). Most of the proteins are predicted to be either GPI-anchored proteins, to contain a signal peptide or to possess other membrane domains, according to preGPI, signalP and TMHMM website, respectively. Six protein families appeared to be typical of Cryptococcus EVs, including the Chitin deacetylase family (Cda), the Ricin-type beta-trefoil lectin domain-containing protein family (Ril), the putative glyoxal oxidase family (Gox), the tetraspanin membrane proteins containing a SUR7/Pall family motif (Tsh), the pr4/barwin domain protein family (Blp), and the multicopper oxidase (Cfo). Among these families, the proteins present in all three species are shown in green, proteins 
1130

1131

1132

1133

1134

1135

1136

1137

1138

1139

1140

1141

1142

1143

1144

1145

1146

1147

1148

1149

1150

1151

1152

1153

1154

1155

1156

1157

1158

1159

1160

1161

1162

present in two species in orange and proteins present in only one species in yellow (C). We also identify 21 putative GPI-anchored proteins, as predicted by PredGPI, and 10 of them were present in all three species (D).

Figure 6. Flow cytometry analysis of $C$. neoformans EVs incubated with GFP-labelled ConA.

FACS analysis of EVs obtained from C. neoformans wild type and cap59A cells. EVs were incubated with ConA-Alexa Fluor 488 conjugated lectin. After ultracentrifuge washing, the EV pellets were mixed in BD Trucount tubes (BD Biosciences), containing a known number of fluorescent beads as internal control. The number of events for each reading was fixed to 100,000 events and the percentage and intensity of ConA labeling were recorded. This experiment was repeated three times with similar results.

\section{Figure 7. EV proteinase $\mathrm{K}$ treatment reduces ConA binding.}

FACS analysis of EVs obtained from C. neoformans WT and cap59A cells after proteinase K treatment. Proteinase K-treated EVs were submitted to ConA labelling, ultracentrifuge washed and analyzed by flow cytometry. EV pellets were mixed in BD Trucount tubes (BD Biosciences), containing a known number of fluorescent beads as an internal control. The number of events for each reading was fixed to 100,000 events and the percentage and intensity of ConA labeling were recorded. EVs treated using the same protocol but omitting the enzyme were used as controls.

\section{Figure 8. Analysis of C. neoformans mutant strain EVs.}

Evaluation of EV production by the different mutant strains as estimated by the measure of the sterol concentration using the Amplex ${ }^{\mathrm{TM}}$ Red Cholesterol Assay Kit (A). Impact of the different mutations on the percentage of ConA-labelled EVs as estimated through flow cytometry (B). Analysis of EV size diameter in the mp88A and alg34 mutant strains as compared to the wild type (WT). The total number of vesicles analyzed were WT ( $\mathrm{n}=143$ for size and $\mathrm{n}=112$ for decoration), $m p 88 \Delta(\mathrm{n}=107$ for size and $\mathrm{n}=86$ for decoration), alg3A ( $\mathrm{n}$ $=119$ for size and $\mathrm{n}=92$ for decoration) (C). Analysis of the decoration thickness revealed a smaller distribution associated with $A L G 3$ or MP88 deletions, as exemplified by illustrative images from the three strains (D). The cryo-EM images were obtained using a TECNAI F20 transmission electron microscope. ConA labelling and sterol measurements were done for at 
1163 least three independent biological replicates Error bars are represented as means \pm SD. Scale

1164 bars represent $100 \mathrm{~nm}$ in $\mathrm{C}$ and $20 \mathrm{~nm}$ in D. (E)

1165

Figure 9: Model of simplified molecular structure and composition of Cryptococcus EVs.

1167 In accordance with previous reports and in the light of our data, a new model of Cryptococcus

1168 EVs is suggested, where the outer layer is composed of the capsular polysaccharide

1169 glucuronoxylomannan (GXM), and the lipid bilayer is covered by many proteins, including

1170 mannoproteins, making the visible fibrillar structure resolved by cryo-EM. Most of the

1171 proteins are predicted to be GPI-anchored, to contain a signal peptide or to possess other

1172 membrane domains, according to preGPI, signalP and TMHMM, respectively. Three proteins,

1173 the hypothetical protein Cpc1, the putative V-type ATPase (Vma10) and the Vep3 are

1174 predicted to be soluble. It is still unclear if these proteins are indeed inside the vesicular lumen

1175 or linked to another transmembrane protein. For simplification, the lipid content was not

1176 explored, but previous works shown the presence of sterol, phospholipoids and sphingolipids.

1177 Additionally, Cryptococcus EVs were also described to contain other cargoes, such as RNA,

1178 pigments, small molecules, and polysaccharides, including GXM, as detailed in plain text.

1179

1180 Figure 10. Vaccination assays using $C$. neoformans EVs.

1181 Female 6-weeks old BALB/c mice were immunized with $C$. neoformans EVs via intraperitoneal injection, followed by intranasal infection with $1 \times 10^{4}$ yeasts of wild-type (WT) C. neoformans, and the mouse survival was monitored. In the first pilot experiment, mice ( $\mathrm{n}=4$ per group) were immunized with EVs from wild type or cap594 strain (1 and 10 $\mu \mathrm{g}$ in $100 \mu \mathrm{L}$ of PBS) and control mice were injected with $100 \mu \mathrm{L}$ PBS. Western Blot using mouse sera against fungal EV confirmed that all immunized mice produced antibodies against EV proteins (A). All EV-immunized mice survived longer than the non-immunized ones, but the immunization with cap594 EVs rendered a significantly prolonged mouse survival $(* p=$ 0.01) (B). For the second set of experiment, mice ( $n=10$ per group) were immunized with EVs from cap594 mutant strain $(10 \mu \mathrm{g} / 100 \mu \mathrm{L}$ in PBS $)$ and control mice were injected with $100 \mu \mathrm{L}$ PBS. Again, Western blot using mouse sera against fungal EVs confirmed that all immunized mice produced antibodies against EV proteins $(\mathrm{C})$. EV-immunized mice showed significantly prolonged survival $(* p=0.0006)$ compared to the non-immunized group (D). 
bioRxiv preprint doi: https://doi.org/10.1101/2020.08.17.253716; this version posted May 17, 2021. The copyright holder for this preprint (which was not certified by peer review) is the author/funder, who has granted bioRxiv a license to display the preprint in perpetuity. It is made available under aCC-BY-NC-ND 4.0 International license.

1197 


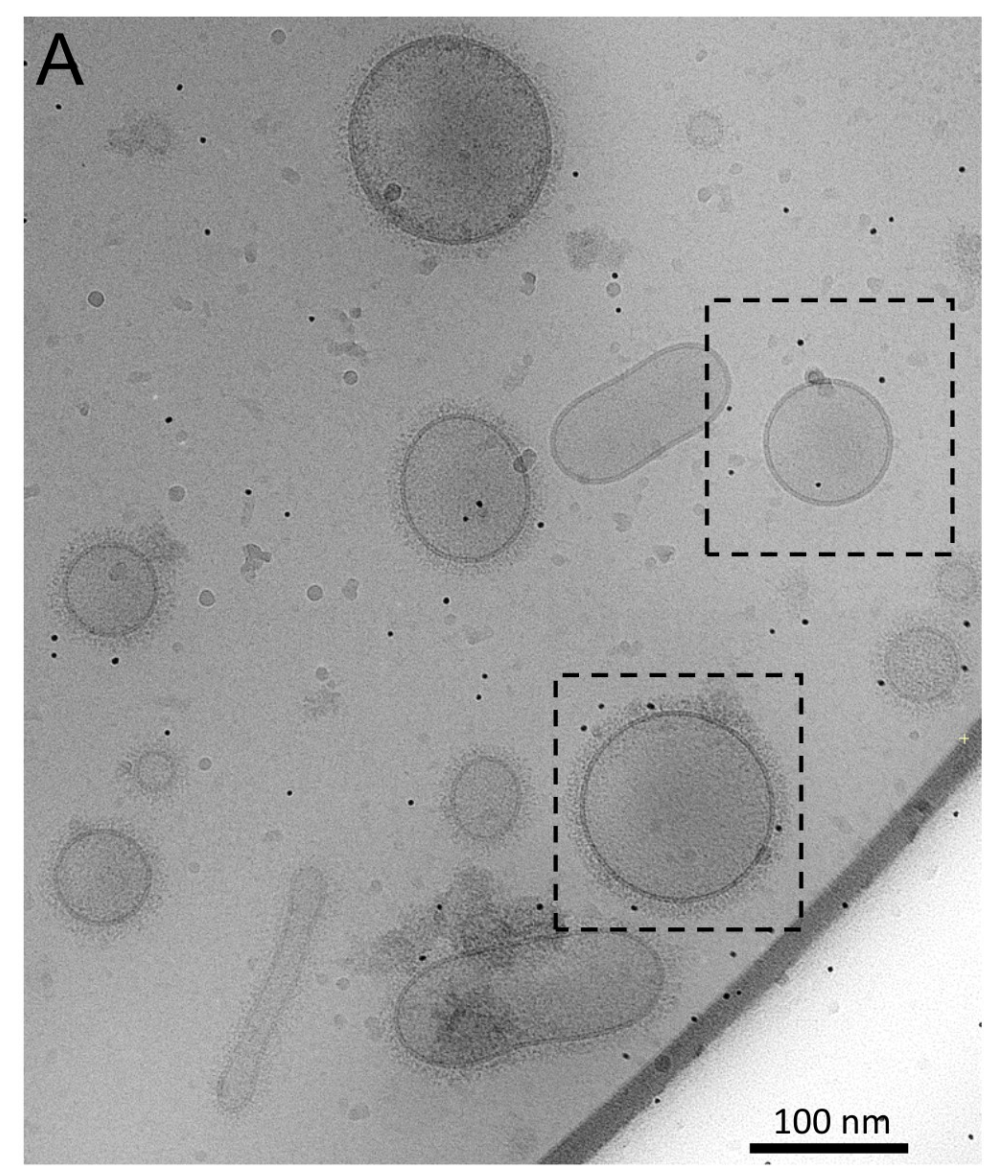

Non-decorated EVs: $10.8 \%$

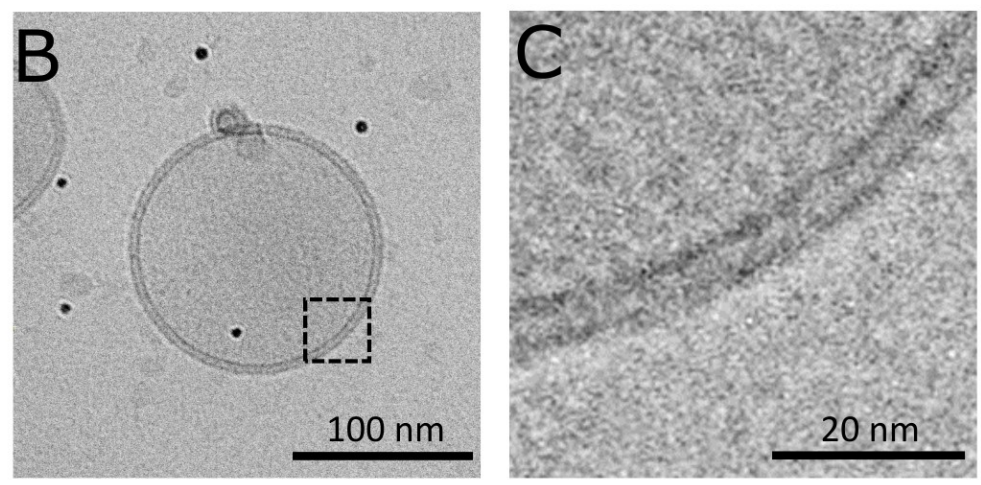

Decorated EVs: $89.2 \%$
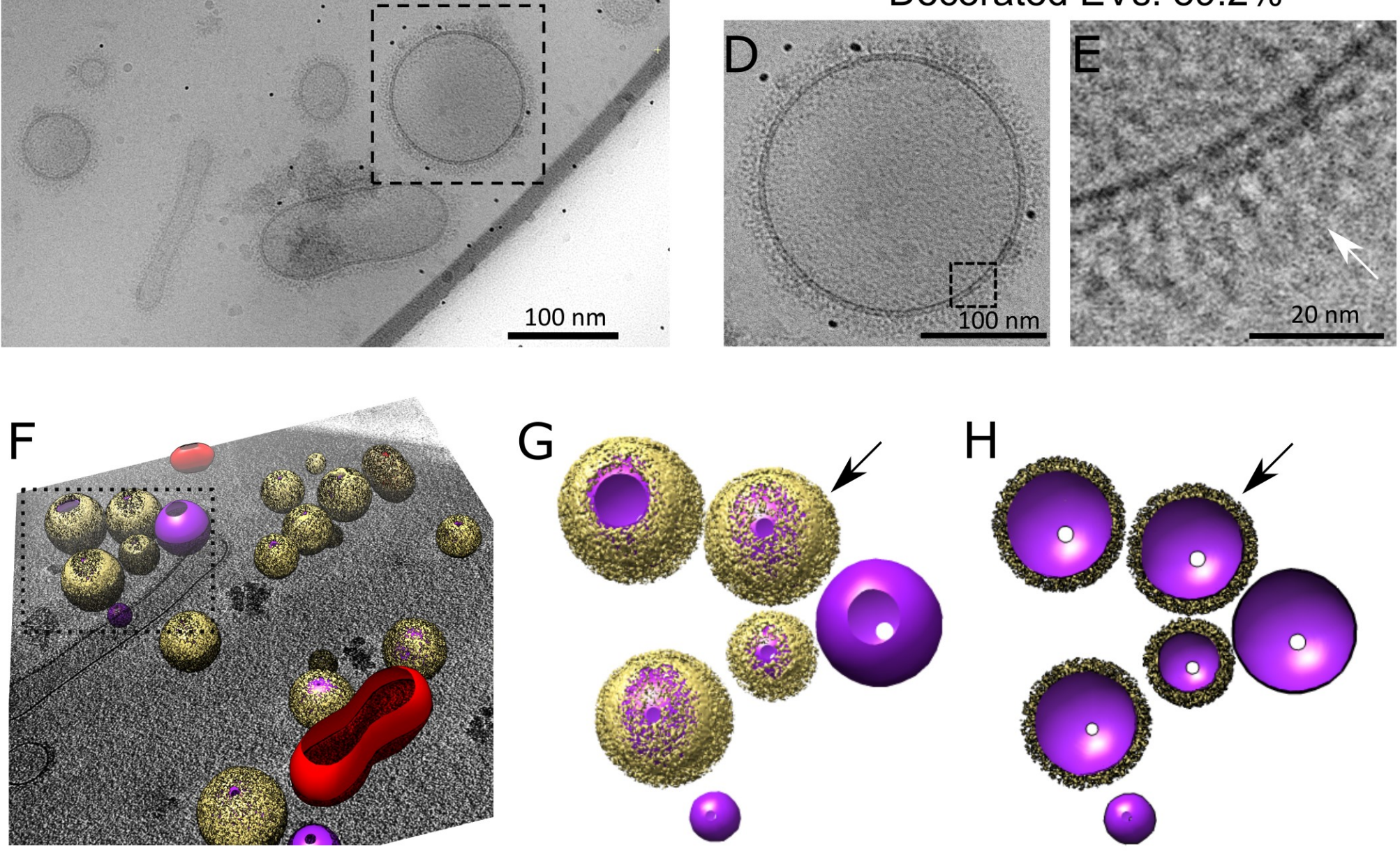


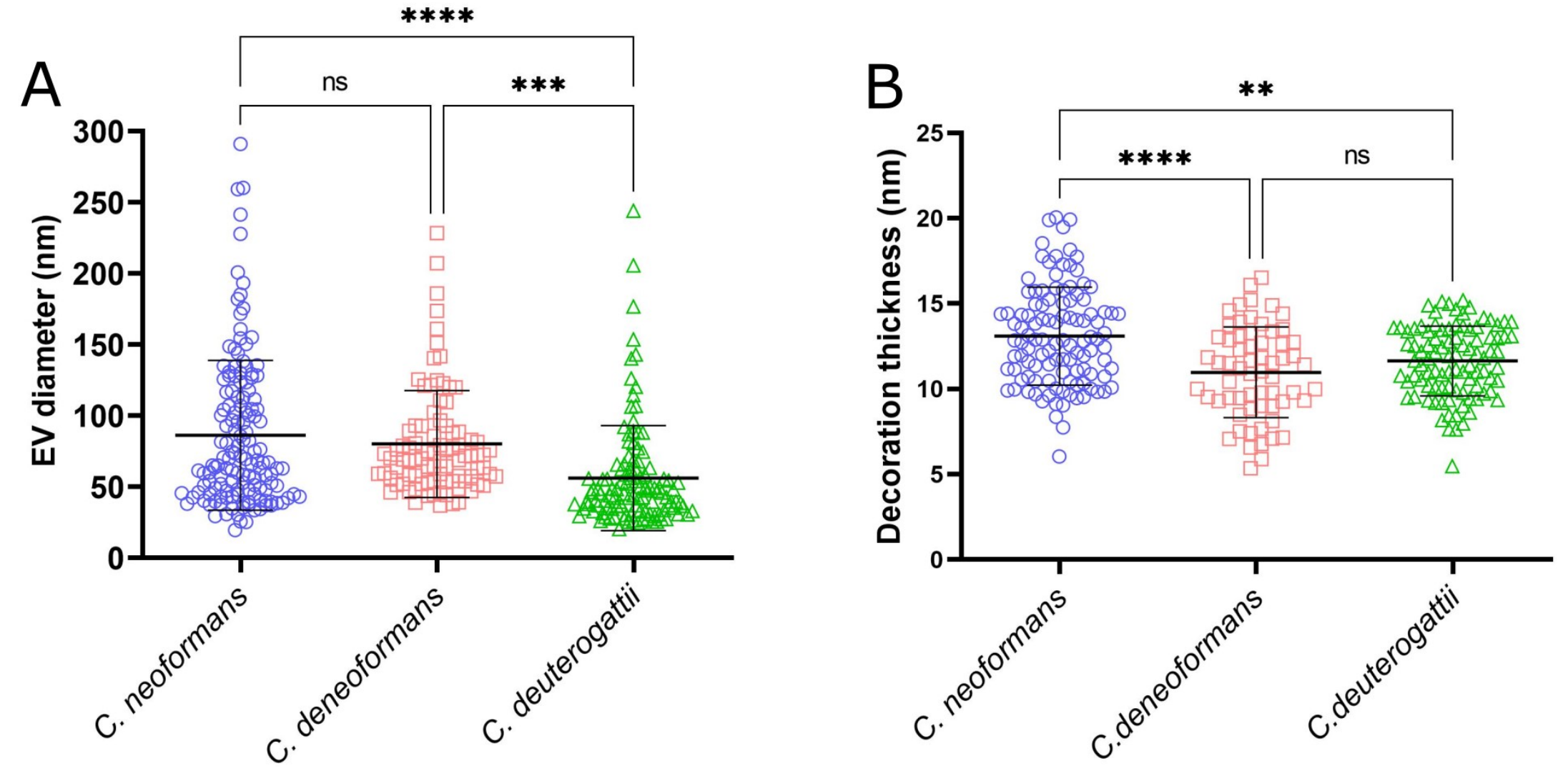

C C. neoformans

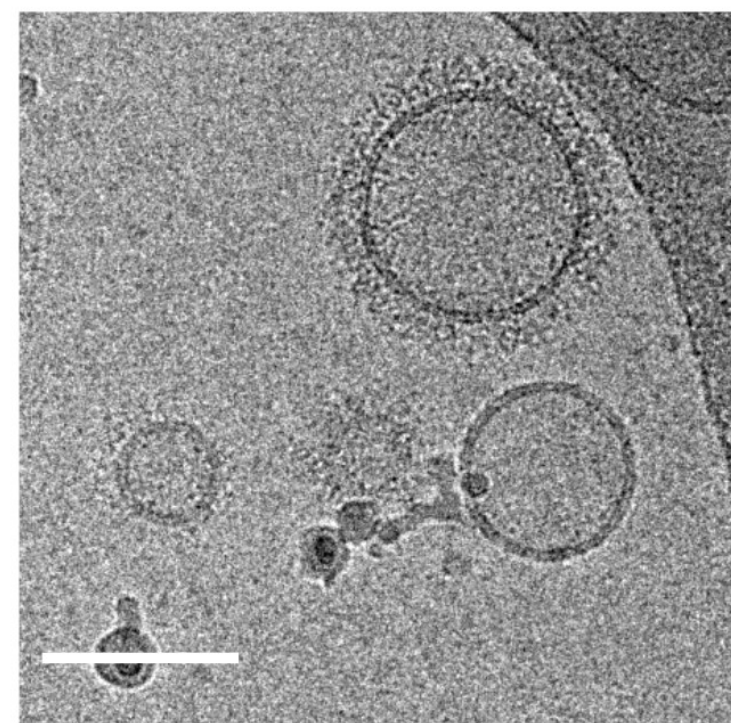

C. deneoformans

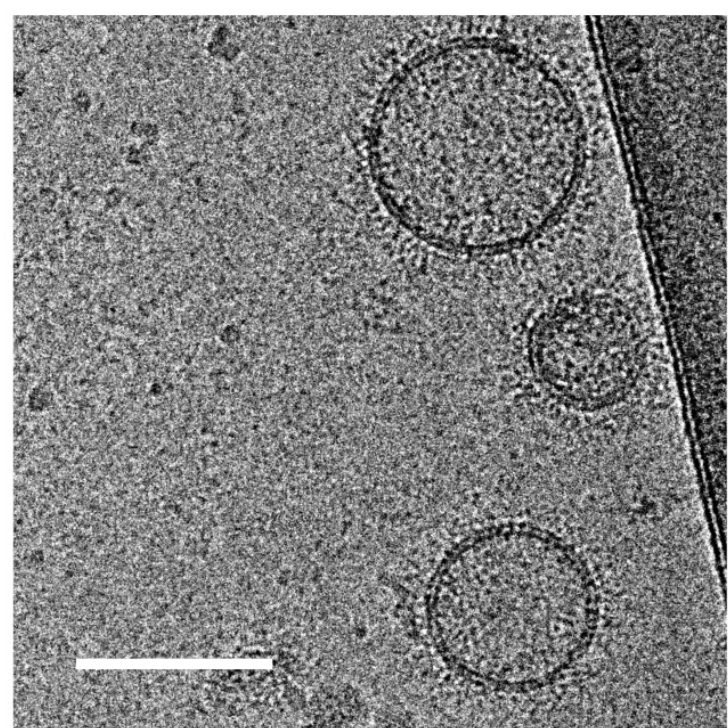

C. deuterogattii

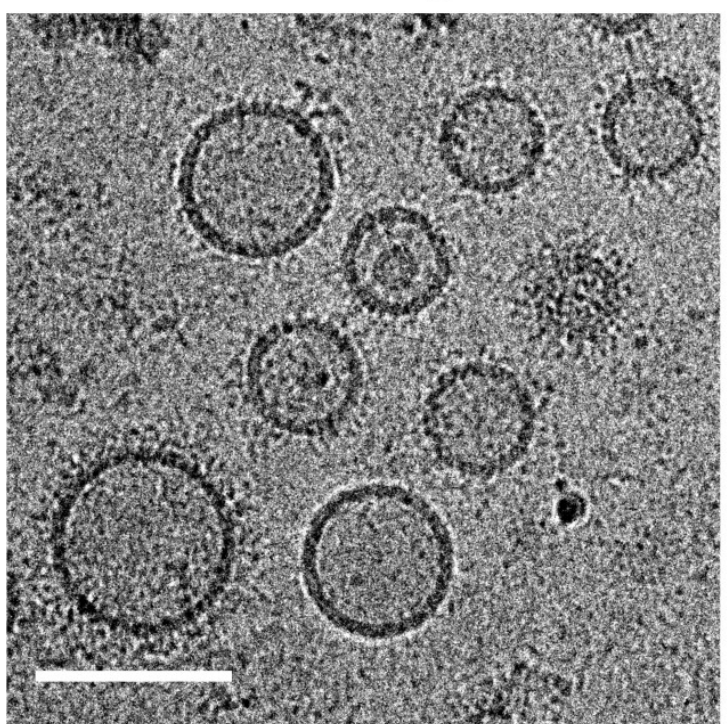


bioRxiv preprint doi: https://doi.org/10.1101/2020.08.17.253716; this version posted May 17, 2021. The copyright holder for this preprint (which was not certified by peer review) is the author/funder, who has granted bioRxiv a license to display the preprint in perpetuity. It is made available under aCC-BY-NC-ND 4.0 International license.
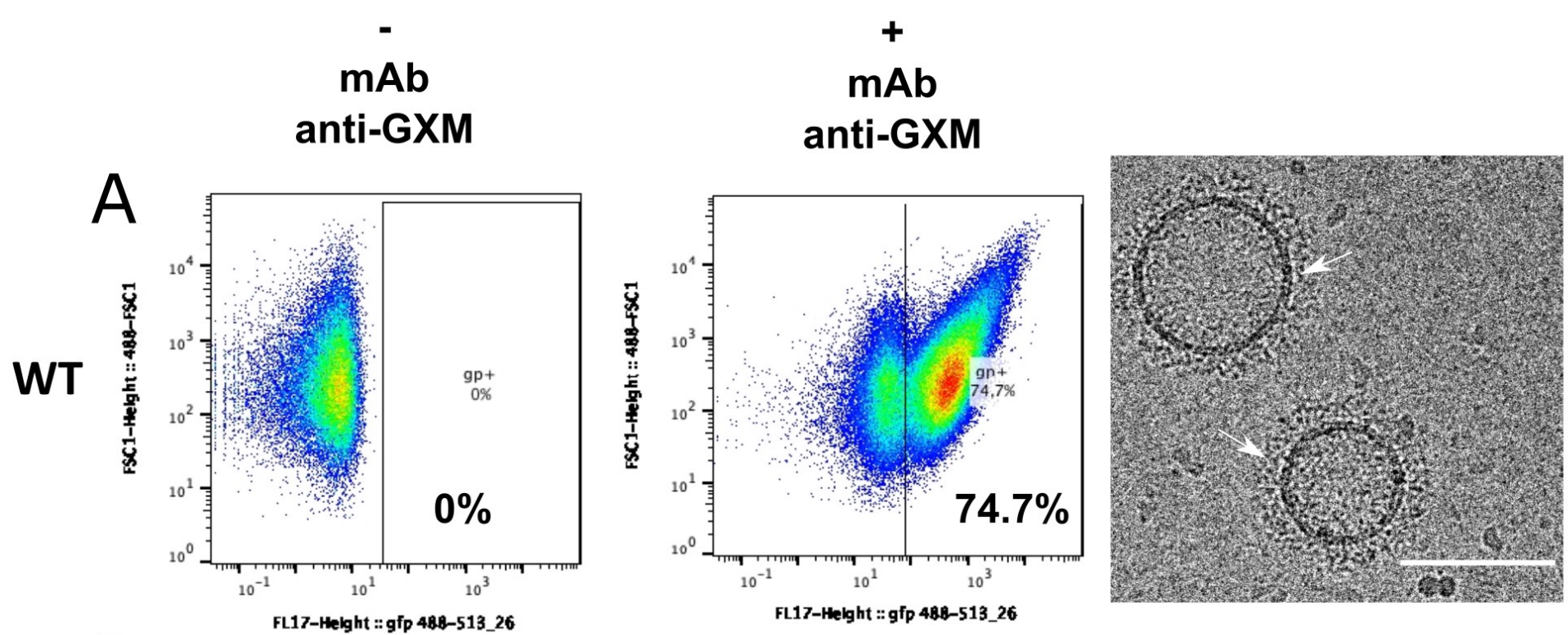

B
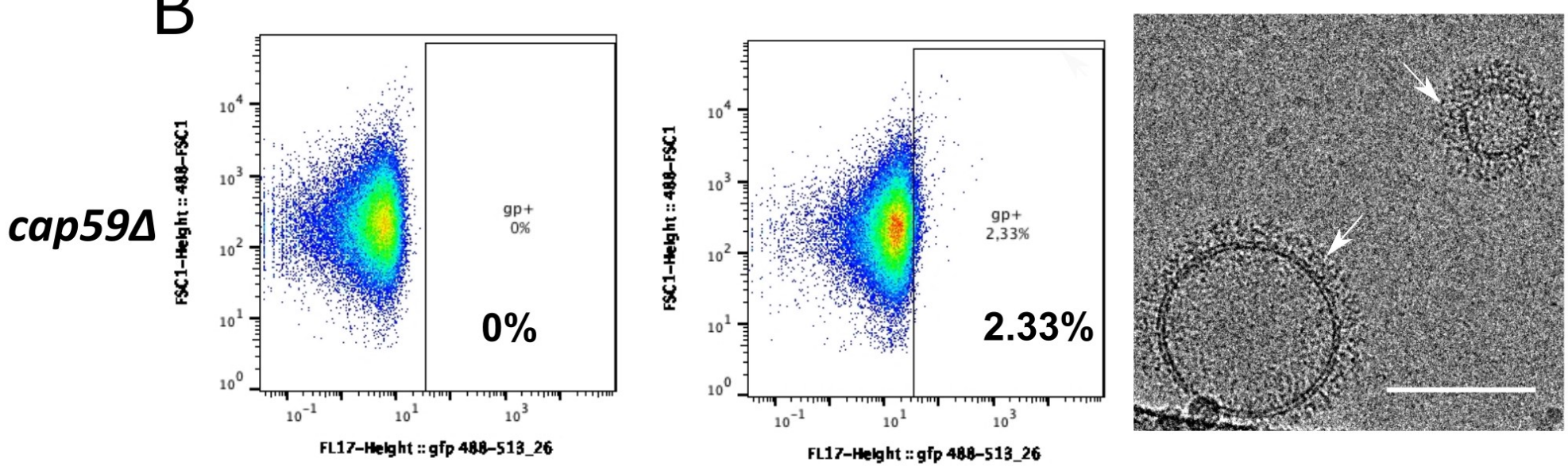

\section{C. albicans}

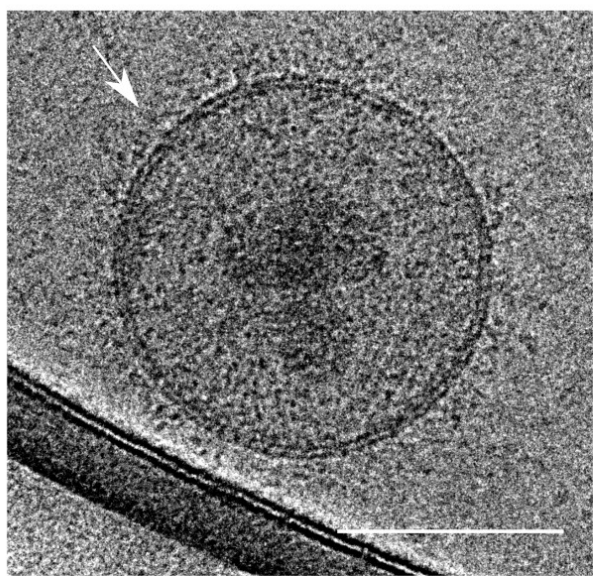

S. cerevisiae

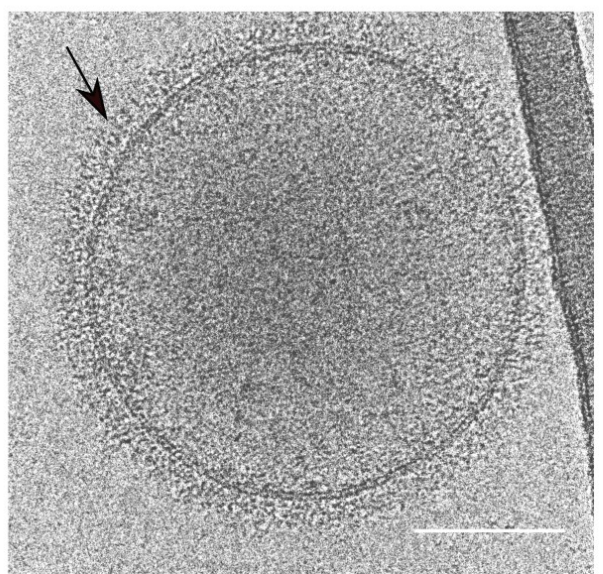


A

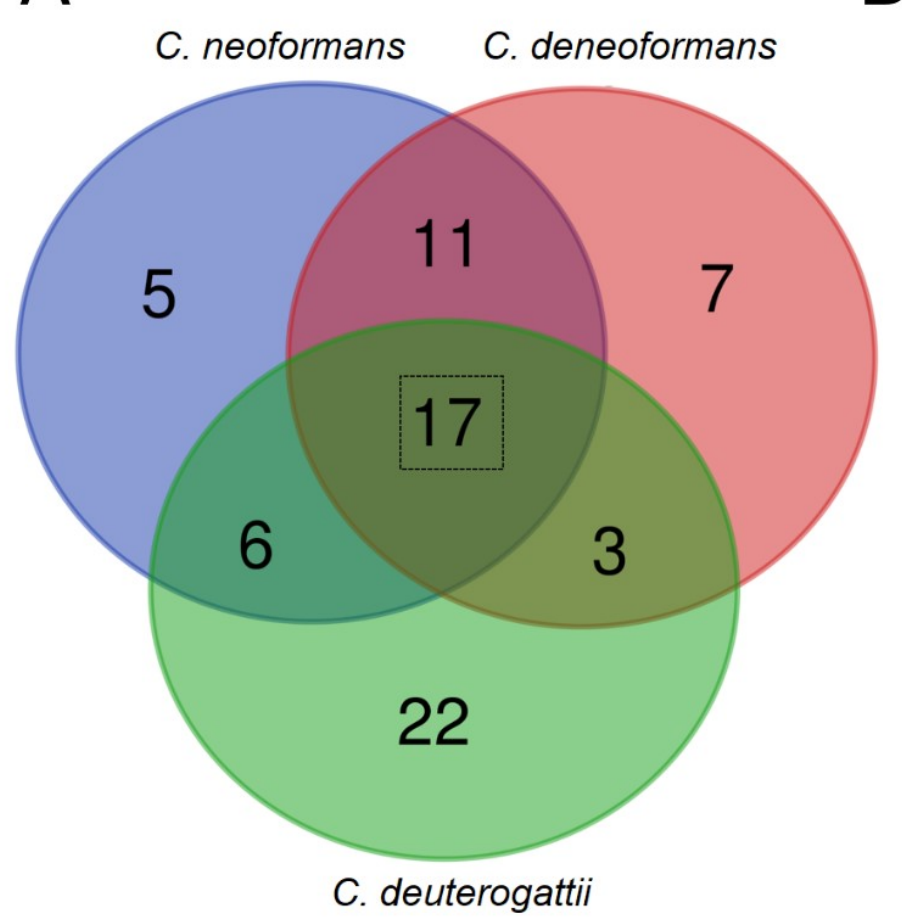

C

\begin{tabular}{|c|c|c|c|c|c|}
\hline Cda1 & Ril1 & Gox1 & Tsh1 & \multicolumn{2}{l}{} \\
Cda2 & Ril2 & Gox2 & Tsh2 & Blp2 & Cfo1 \\
\cline { 1 - 1 } Cda3 & Ril3 & Gox3 & Tsh3 & Blp4 & Cfo2 \\
Cda & Ril & Gox & Tsh & Blp & Cfo \\
\hline
\end{tabular}

Protein families
List of EV- enriched proteins shared by the three Cryptococcus spp.

\begin{tabular}{lllll}
\hline C. deuterogattii & C. deneoformans & C.neoformans & Protein name & \\
\hline CNBG_1155 & CNA07540 & CNAG_00776 & Mp88 & Immunoreactive mannoprotein \\
CNBG_3648 & CNC06180 & CNAG_03007 & Cpc1 & DUF3759 \\
CNBG_3432 & CNC03950 & CNAG_02775 & Bim1 & BCS-inducible membrane protein \\
CNBG_9064 & CND03490 & CNAG_01230 & Mp98/Cda2 & Chitin deacetylase \\
CNBG_5182 & CNE05040 & CNAG_02030 & Gox2 & Glyoxaloxidase \\
CNBG_3374 & CNC03380 & CNAG_01854 & Hep2 & Heparinasell/II family protein \\
CNBG_1745 & CNF01800 & CNAG_05799 & Cda1 & Chitin deacetylase \\
CNBG_4970 & CNN02260 & CNAG_06501 & Gas1 & 1,3-Beta-glucanosyltransferase \\
CNBG_5817 & CNJ03160 & CNAG_04891 & Ril1 & Ricin-type beta-trefoil lectin domain-containing \\
PNBG_4258 & CNH02560 & CNAG_05615 & Sso1 & t-SNARE complex subunit \\
CNBG_9173 & CNF01900 & CNAG_05788 & Vma10 & V-type ATPase, G subunit \\
CNBG_5332 & CNE03480 & CNAG_02189 & Amy1 & Alpha-amylase \\
CNBG_4145 & CNE01150 & CNAG_02443 & Vep3 & NADH dehydrogenase \\
CNBG_5365 & CNE03150 & CNAG_02225 & Exg104 & Glucan 1,3-beta-glucosidase \\
CNBG_0679 & CND02350 & CNAG_01109 & Vep4 & Hypotheticalprotein \\
CNBG_0806 & CND03580 & CNAG_01239 & Cda3 & Chitin deacetylase \\
CNBG_5038 & CNN01470 & CNAG_06422 & Vep5 & Hypotheticalprotein \\
\hline & & & &
\end{tabular}

D

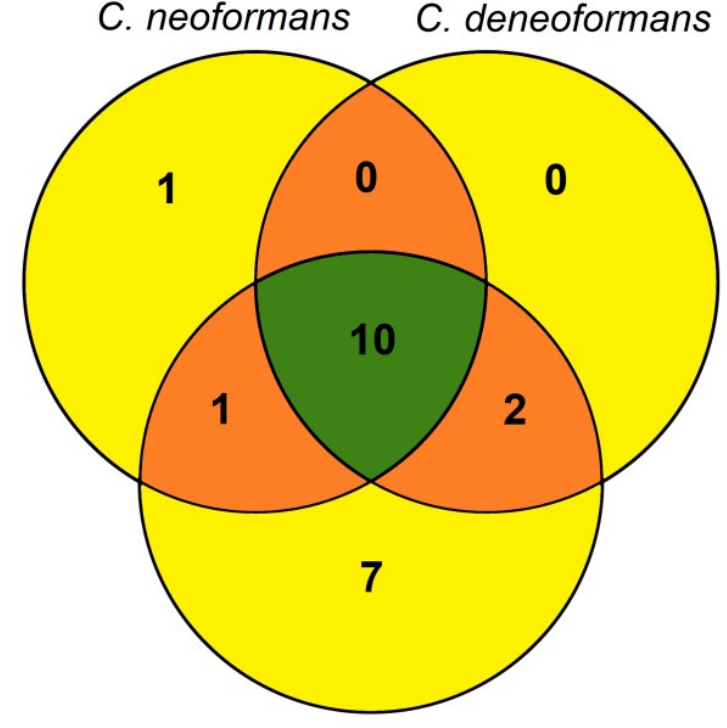

C. deuterogattii 
bioRxiv preprint doi: https://doi.org/10.1101/2020.08.17.253716; this version posted May 17, 2021. The copyright holder for this preprint (which was not certified by peer review) is the author/funder, who has granted bioRxiv a license to display the preprint in perpetuity. It is made available under aCC-BY-NC-ND 4.0 International license.
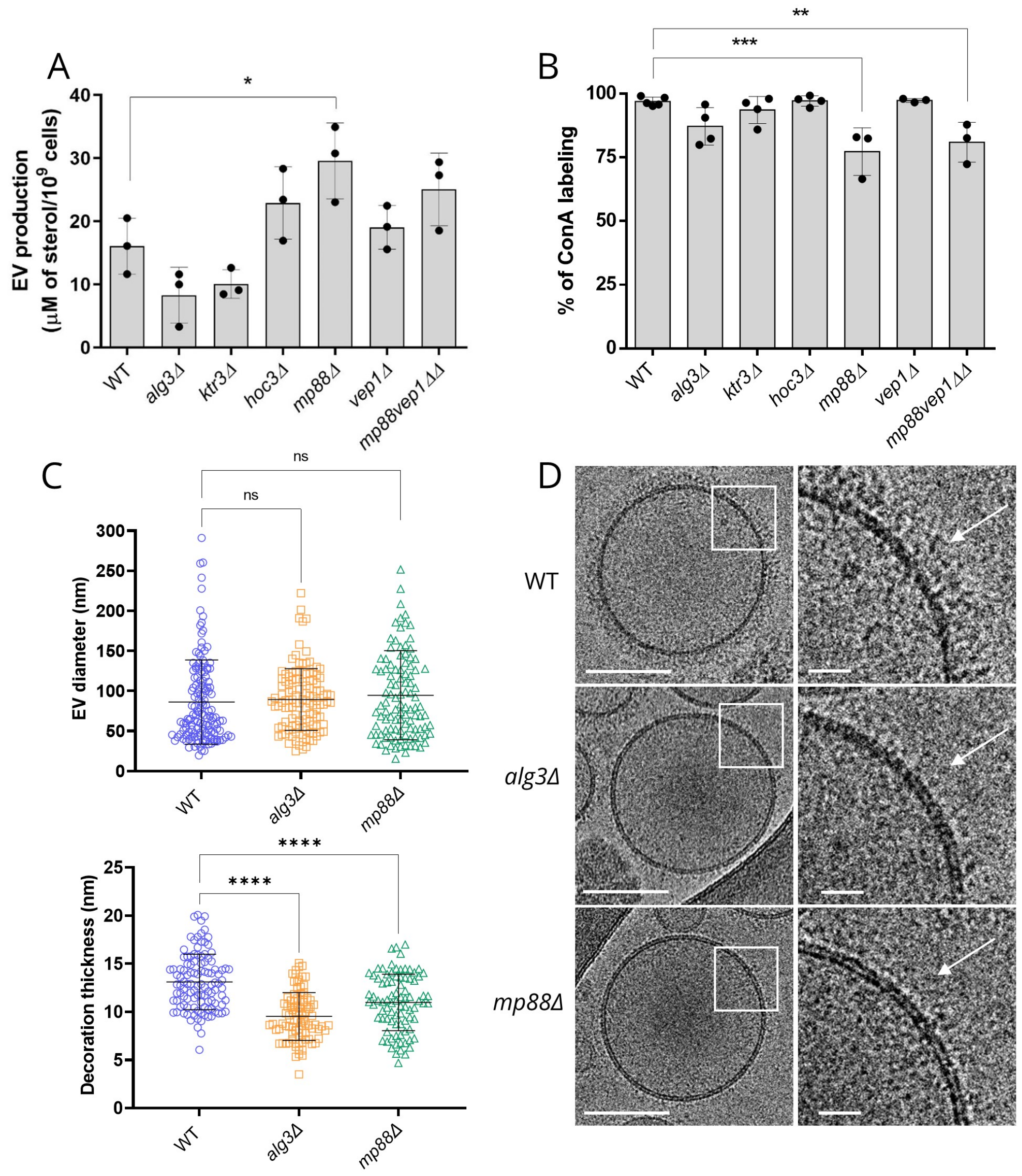


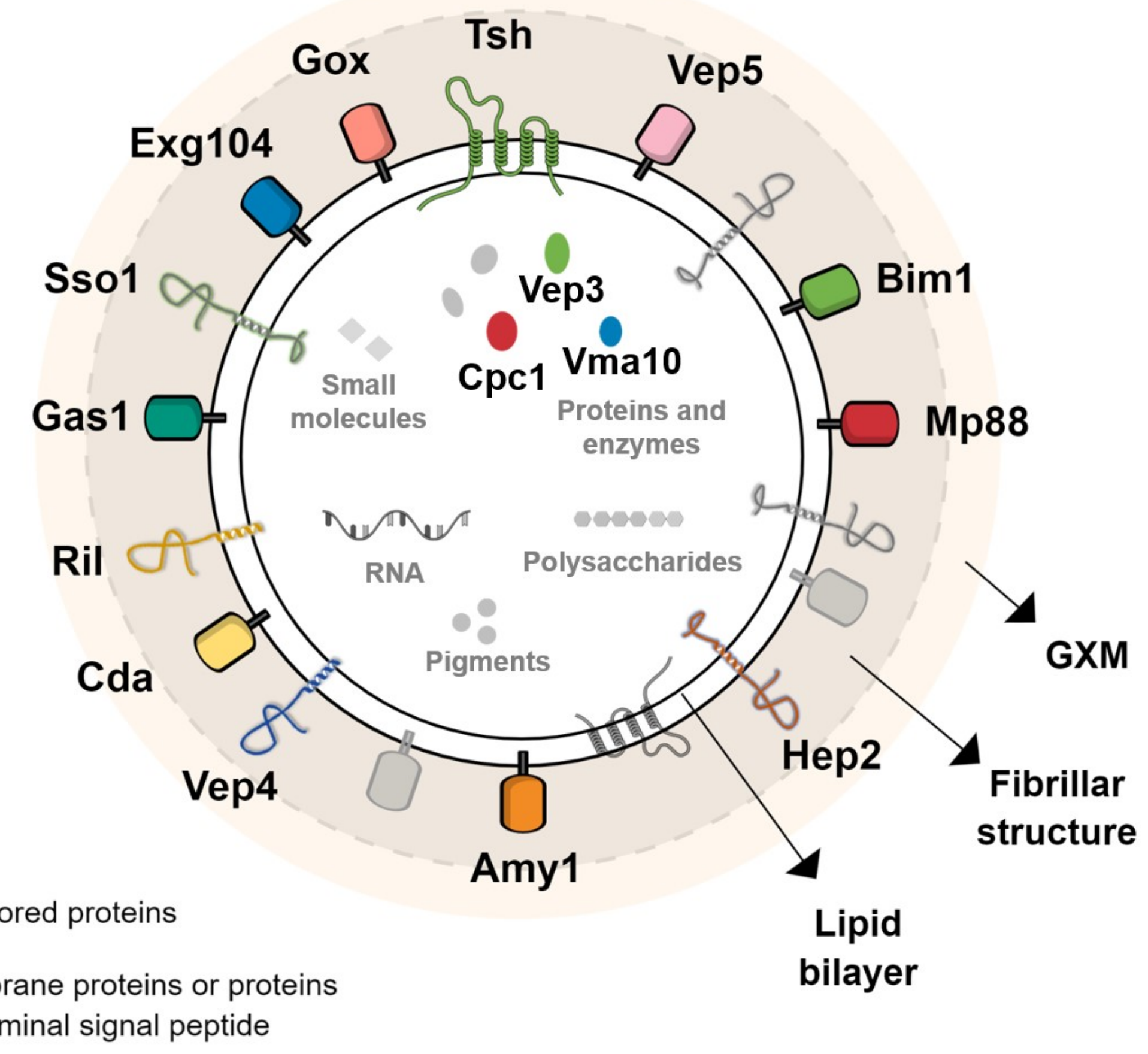


A

Immunized mice
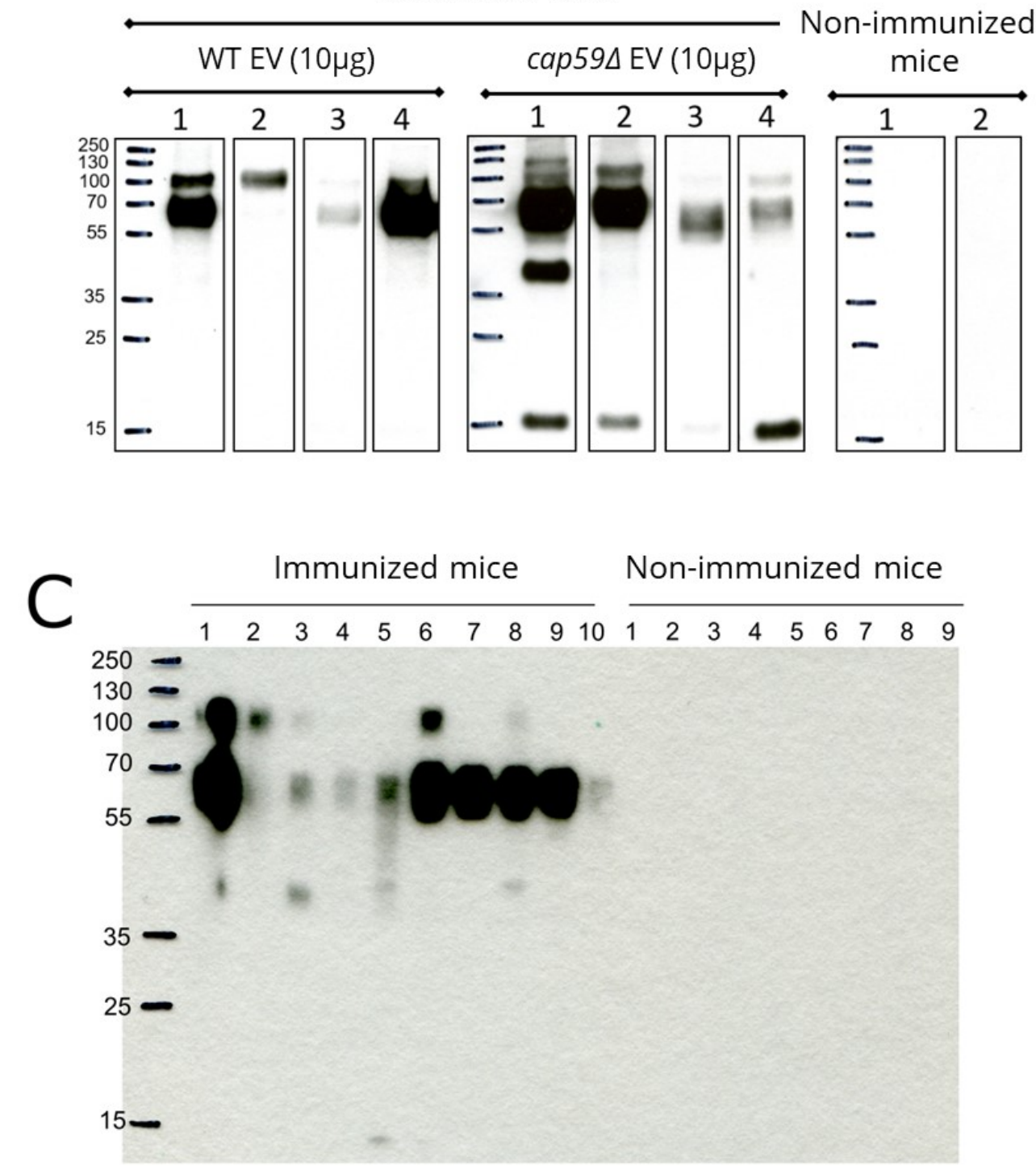

B

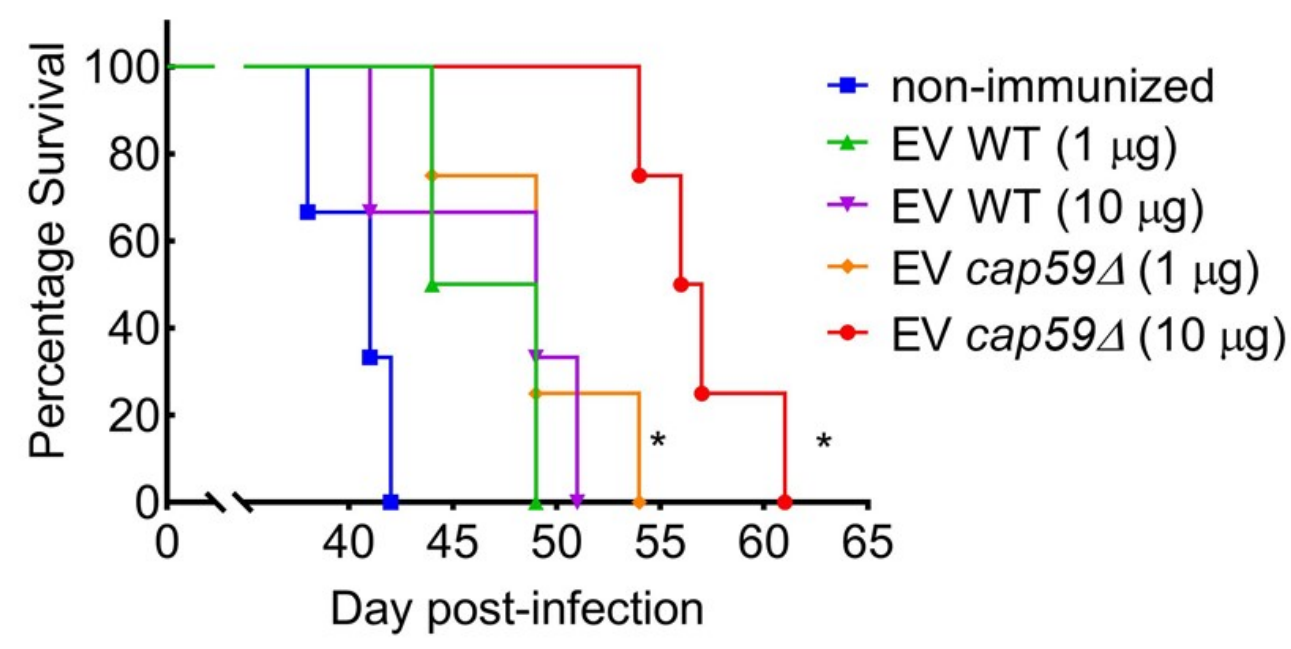

D

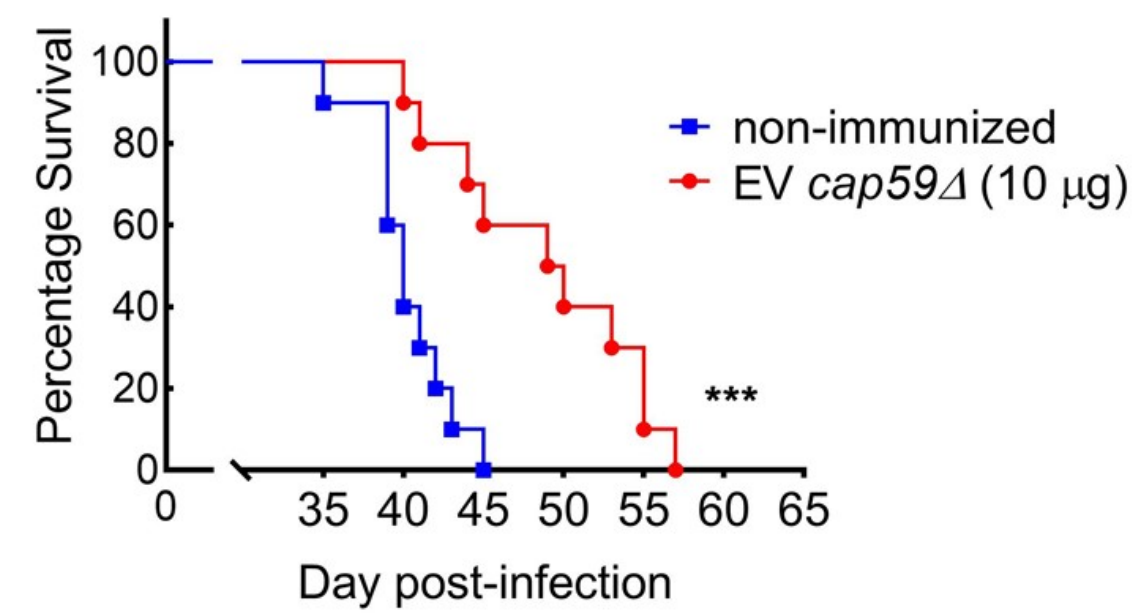

